Article

\title{
Improving Reliability and Energy Efficiency of Three Parallel Pumps by Selecting Trade-Off Operating Points
}

\author{
Safarbek Oshurbekov, Vadim Kazakbaev (D), Vladimir Prakht* (D) and Vladimir Dmitrievskii (D) \\ Department of Electrical Engineering, Ural Federal University, 620002 Yekaterinburg, Russia; \\ safarbek.oshurbekov@urfu.ru (S.O.); vadim.kazakbaev@urfu.ru (V.K.); vladimir.dmitrievsky@urfu.ru (V.D.) \\ * Correspondence: va.prakht@urfu.ru; Tel.: +7-343-375-45-07
}

\begin{abstract}
Reliability, along with energy efficiency, is an important characteristic of pump units in various applications. In practical pump applications, it is important to strike a balance between reliability and energy efficiency. These indicators strongly depend on the applied control method of the pump unit. This study analyzes a trade-off method for regulating a system with three parallel pumps equipped with only one frequency converter (multi-pump single-drive system). A typical operating cycle of a pumping system with variable flow rate requirements is considered. The proposed trade-off method is compared with the traditional regulation, when a change in the operating point of the pump is achieved only by changing the rotation speed, and with the method for maximum reliability. It is shown that the proposed trade-off method makes it possible to ensure sufficient reliability of the multi-pump system operation without a significant increase in energy consumption.
\end{abstract}

Keywords: centrifugal pump; energy efficiency; induction motor; parallel pumps; throttling; variable speed pump

Citation: Oshurbekov, S.; Kazakbaev,

V.; Prakht, V.; Dmitrievskii, V.

Improving Reliability and Energy

Efficiency of Three Parallel Pumps by Selecting Trade-Off Operating Points. Mathematics 2021, 9, 1297.

https://doi.org/10.3390/math9111297

Academic Editor: Nicu Bizon

Received: 5 April 2021

Accepted: 3 June 2021

Published: 5 June 2021

Publisher's Note: MDPI stays neutral with regard to jurisdictional claims in published maps and institutional affiliations.

Copyright: (c) 2021 by the authors. Licensee MDPI, Basel, Switzerland. This article is an open access article distributed under the terms and conditions of the Creative Commons Attribution (CC BY) license (https:// creativecommons.org/licenses/by/ $4.0 /)$.

\section{Introduction}

Pumps consume about $20 \%$ of the electricity generated worldwide [1]. Most of the life cycle cost of a pump is the cost of the electricity it consumes. Therefore, pumps are one of the most promising applications for the implementation of energy-saving technologies. At the same time, maintenance and repairs account for a significant part of the life cycle costs of a pump unit (Figure 1a). Therefore, when optimizing the total life cycle costs, in addition to the initial cost of equipment and electricity costs, it is necessary to take into account also the costs of maintenance and repairs, which are affected by the reliability of the pump [2]. It was shown in [3,4] that mean time between failure (MTBF) can be used to quantify pump reliability. In turn, MTBF depends on the deviation of the pump flow rate Q from the Best Efficiency Point (BEP, Figure 1b) [5,6].

Parallel pumps are widely used in many applications, such as when high flow rates or wide flow control ranges are required. When using parallel pumps, it is possible to significantly reduce all components of the life cycle cost of a pumping station, in comparison with a single-pump unit of the same rated power [3,7]. At the same time, due to a large number of variable parameters and the nonlinearity of such systems, the problems of optimizing the energy consumption of parallel pumps, taking into account the reliability and cost of the life cycle, are complex and still not considered very often in the literature.

Many studies analyze the energy consumption of parallel pumps, without taking into account their reliability. Thus, in [8], parallel operation of two pumps with the same rated head and different rated flow is investigated. It is shown that the overall efficiency of the system decreases with an increase in the ratio of the rated flow rates of individual pumps. This study compared the performance of 28 different combinations of the pumping system parameters. In [9], parallel operation of a multi-pump system consisting of four pumps is considered, in which the flow is regulated by three different methods (throttling, bypass 
and speed control). A genetic algorithm and various optimization criteria were used to find the most economical control strategy. It was shown that the lowest energy consumption is achieved when the rotational speed of all pumps is simultaneously controlled at the same value. In [10], an optimization of the total cost of systems of three parallel and three serial pumps without rotational speed control is considered, taking into account the cost of the pump, pipeline and energy consumption. In [11], an optimization of energy consumption of a system of seven parallel pumps with various ratings, some of which are equipped with variable speed drive (VSD), is considered. In [12], energy consumption of three parallel pumps when changing the number of VSDs is compared. In [13], energy consumption of pumping systems with 2-4 parallel pumps without speed control is compared. In [14], a predictive control algorithm is proposed for a system of 3 parallel pumps, each of which is equipped with a VSD, which allows, based on look-up tables, to increase the system efficiency.

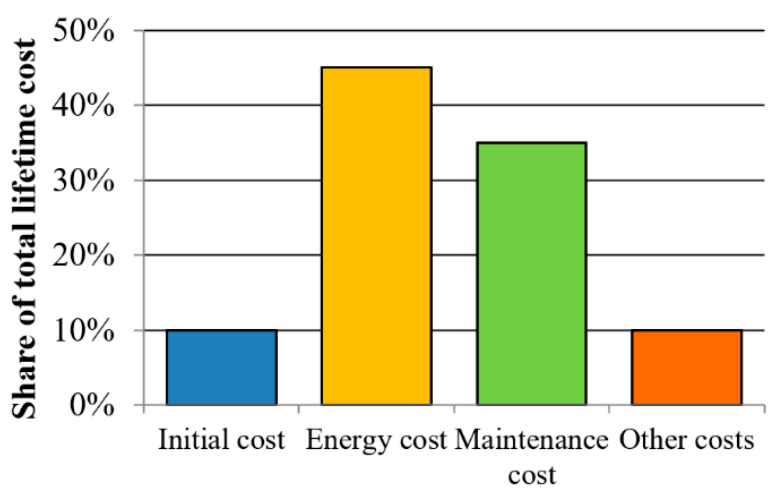

(a)

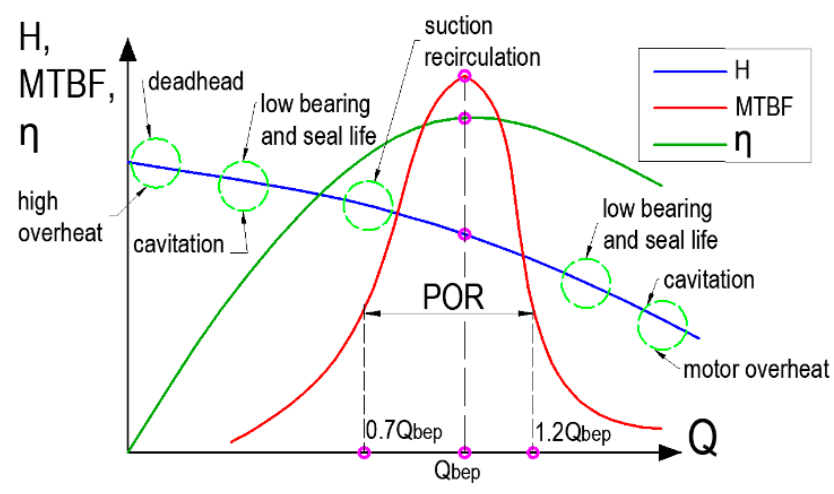

(b)

Figure 1. Pump statistics: (a) Components of the pump lifetime cost [2]; (b) Pump head (H), reliability (MTBF) and efficiency

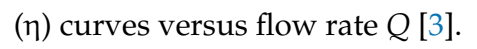

Several works on parallel pumps consider not only energy consumption, but also the deviation of the pump operating points from the BEP. In [15], an optimization of the energy consumption of a system of two parallel pumps using a genetic algorithm is considered. The results show that the lowest energy consumption of the system is obtained by equalizing the flows of the two pumps. Furthermore, [15] compares the energy consumption of the two pumps equipped with a different number of VSD: one or two. It is shown that in the latter case, it is possible to achieve a lower energy consumption. The deviation of the operating point of the pumps from the BEP in various considered cases is compared, but not optimized.

In [6], an optimization of a single-pump unit is considered to increase its reliability using a genetic algorithm. However, some aspects were not taken into account: the optimization criterion is only the maximum reliability of the pump unit. Achieving a trade-off between reliability and power consumption is not considered. The study also does not consider the use of bypass regulation to obtain a better operating point. The static head in the hydraulic system is assumed to be zero, which also reduces the range of cases to which the results of this study are applicable.

In [16], a control strategy is proposed for a variable speed multi-pump system to reduce energy consumption. It was shown that the system of parallel pumps has the highest efficiency when the pumps operate at the same speed and flow rate than when one of the pumps operates at the rated speed and the speed of the other one is adjusted to obtain the required flow rate. It is also shown that in the former case, the deviation of the pump operating point from the BEP is less.

In [17], an analysis of a single-pump unit with a power rating of $11 \mathrm{~kW}$ is carried out and the issues of reducing energy consumption and increasing reliability are investigated. 
A trade-off regulation is proposed that provides good pump reliability with the energy consumption close to the minimum. The energy consumption is calculated for three cases: the conventional rotational speed regulation, regulation with maximum reliability and trade-off regulation. It is shown that when using the trade-off regulation, it is possible to significantly reduce the energy consumption of the pump unit in comparison with the case of maximum reliability, while maintaining all pump operating points in the preferred operating range with sufficient reliability.

In [3], an optimal trade-off control method using particle swarm optimization is proposed for a system of two identical parallel pumps, each equipped with a frequency converter (FC). The optimization parameters are the number of simultaneously operating pumps and the rotational speed of the pumps. The optimization criterion is the minimization of energy consumption. The deviation of the pump operating point from the BEP is used as an optimization constraint. It is assumed that both pumps rotate at the same speed at each operating point, and the throttle is used to regulate the flow in the common pipeline section of the parallel pumps. However, the results of this study are only applicable when the speed of both parallel pumps is controlled by VSD. Meanwhile, in practice, parallel pumping systems are often used in which some of the pumps do not have a VSD and are powered directly from the mains [18]. The literature overview carried out shows that not all configurations of parallel pump systems have been analyzed from the point of view of reliability. In particular, multi-motor pumping stations with a single frequency converter (multi-pump single-drive systems), which are also actively used in practice to reduce the total cost of a multi-pump system, were not considered.

This paper analyzes the effectiveness of applying the trade-off regulation method proposed in [17] to a system of 3 parallel pumps. This trade-off method is compared with the traditional regulation and regulation with maximum reliability proposed in [3]. In contrast to [3], a pumping system of the "multi-pump single-drive" type is considered, in which only one frequency converter is used to alternately drive several pump units (in this case, 3 units). Such systems are widely used in parallel pumping stations equipped with low-power electric motors. However, analysis of such systems is not very common in the literature [7].

In such pumping systems, one frequency converter controls two or more pumps. At the same time, in contrast to systems without a frequency converter, a smooth start-up of each pump unit and a smooth flow/pressure adjustment are ensured. In contrast to the case where each pump unit is equipped with an individual frequency converter, the capital cost of the system is significantly reduced. This advantage is especially important if the system uses low-power pump units, for which the cost of the frequency converter is the largest part of the total cost, as well as in systems containing a large number of pumping units $[18,19]$.

Since in multi-pump single-drive systems it is possible to control the rotational speed of only one pump at a time, the load range of the pumps in such a system is significantly different from the case when each of the pumps is equipped with a VSD. Therefore, the conclusions carried out in [3] cannot be directly applied to the multi-pump single-drive systems.

Therefore, the contribution of this article is as follows: the effectiveness of the control principle, which provides a trade-off between reliability and energy efficiency, was theoretically verified in the case of a multi-pump single-drive system, which was not covered in previous studies on the topic.

It should be noted that many studies discuss the selection and setting of dynamic controllers for pumping systems [6,20-25]. Several studies show the effectiveness of fractional-order proportional-integral-derivative control (FOPID) [20] for improving the dynamics of complex non-linear pumping systems. Thus, [21] provides a theoretical and experimental comparative study of a simple single-pump system with different types of regulators, including PI, PID and FOPID. Several papers [22,23] show the advantages of the FOPID over conventional PIDs in controlling pumping systems that provide liquid 
level in a connected tank system. Further improvement of pumping system dynamics can be achieved by adding fuzzy logic to the FOPID [24,25].

Our paper does not discuss the selection and setting of dynamic controllers; however, it evaluates the benefits of applying the proposed approach to the selection of operating points for pumps in a multi-pump system. The novelty of the proposed approach is that it considers both the energy efficiency of the system and its reliability. At the same time, it is assumed that a controller is used that provides a satisfactory quality of transients.

Using the traditional mathematical model of parallel pumps and polynomial interpolation of data from the manufacturer's catalogs, the energy consumption and reliability of the considered multi-pump single-drive system using different regulation methods are compared. The conventional rotational speed regulation and the previously proposed method providing maximum reliability [6] are considered. The benefits of using the proposed trade-off regulation method for the considered pumping system are also evaluated.

The aim of this study is to develop a regulation method that increases the reliability of multi-pump single-drive systems, in which, as will be shown below, when using the traditional control method, individual pumps can operate for a long time in conditions that shorten their service life. Reliability issues have already been discussed in detail in the literature for both single pump systems and systems of parallel pumps, each of which is equipped with a frequency converter $[3,6,15]$. The objective of this study is a comparative analysis of a particular case of a pumping system consisting of three parallel low-power pump units, one of which is powered by a frequency converter and the other two are powered directly from the grid, using different control methods.

\section{Mathematical Equations of the System and Methodology of the Study}

The article uses the traditional analytical method of mathematical analysis of pumping systems, based on the calculation of $Q-H$ (head versus flow) and $Q-P$ (mechanical shaft power versus flow) characteristics, to analyze the performance of the pumping system with various regulation methods. The characteristics corresponding to the rated rotational speed are interpolated using polynomials according to the pump datasheet. The characteristics at the arbitrary rotational speed are calculated using the characteristics at the rated speed and the affinity laws [26]. This study examines the change in energy consumption of the considered multi-pump single drive system when applying to it various restrictions on the deviation of its operating points from the BEP curve which is also the maximum reliability curve.

The parallel pump system serves to provide the required flow rate $Q_{r e q}$ with a constant static head $H_{s t}$ in an open hydraulic system from point $A$ to point $B$ (Figure 2) [26].

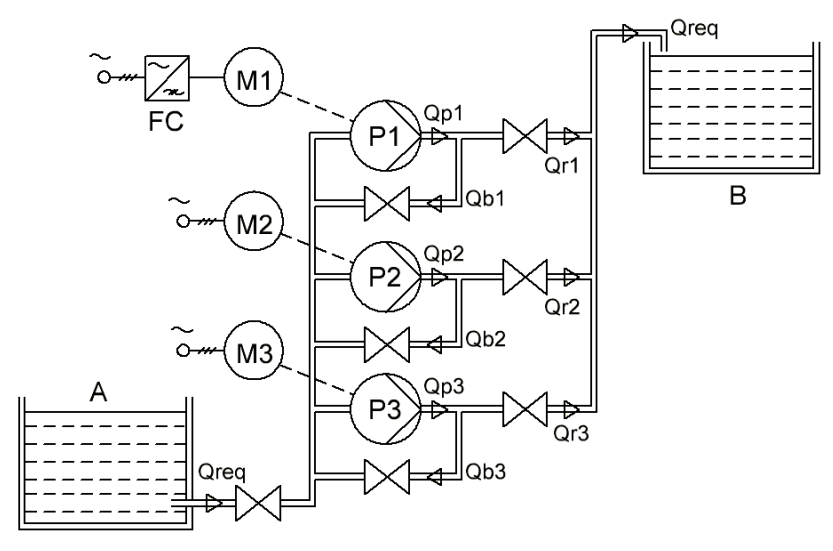

Figure 2. Hydraulic diagram of the pumping system.

Depending on the geometric dimensions of the pipelines (length, section, shape, etc.), the physical properties of the pumped liquid (density, viscosity, etc.) and the difference 
in heights of the basins $A$ and $B$, a curve of the hydraulic system is constructed, which is described by the Equation (1) [26]:

$$
H_{R E Q}=H_{S T}+k \cdot Q_{R E Q}{ }^{2},
$$

where $H_{R E Q}$ is the required hydraulic head; $H_{S T}$ is the static head; $k$ is the hydraulic friction coefficient.

Figure 3 shows the various characteristics of the hydraulic system. Curves labeled with 1 and 2 are the curves of the system at a non-zero value of the static head $H_{S T}$ at different values of $k$. Numbers 3 and 4 mark the curves of the hydraulic system, at different values of $k$ and $H_{S T}=0$. Number 5 marks the dashed curve on which the BEP points lie at different pump speed (BEP curve). The maximum reliability and maximum efficiency of the pump are reached when the system curve matches the BEP curve (dashed curve 5 in Figure 3). The BEP curve is defined according to the affinity laws by the following equation [3]:

$$
H=k_{B E P} \cdot Q^{2},
$$

where $Q_{B E P}$ and $H_{B E P}$ are the flow and the head of the pump at the BEP with $n=n_{\text {rate }}$; $k_{B E P}=H_{B E P} / Q_{B E P} . Q_{B E P}$ and $H_{B E P}$ are defined according to the pump datasheet.

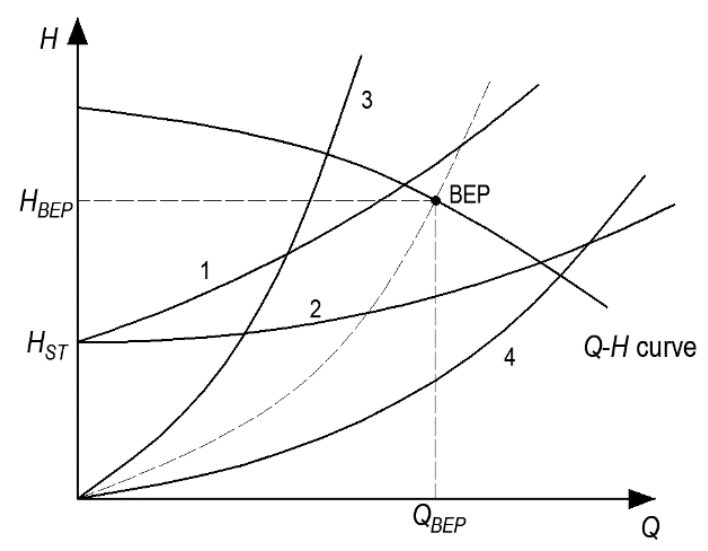

Figure 3. $Q-H$ curve of the pump, hydraulic load curves (marked with numbers 1-4) and BEP curve (marked with number 5).

The selection of the pump unit is carried out based on the maximum values of the required flow rate and head.

In practice, however, they do not match, and the pump operating point may be far from the BEP curve. Therefore, depending on the parameters of the hydraulic load curve (1), it is necessary to shift the pump operating point closer to the BEP curve to increase the pump reliability.

If the hydraulic system curve is located to the right of the BEP curve (as curve 4 in Figure 3), then to increase the pump reliability, it is necessary to regulate the water flow by adjusting the rotational speed and throttling [6]. If the hydraulic system curve is located to the left of the BEP curve (as curve 3 in Figure 3), then bypass also must be applied. However, the use of throttling and bypass along with the regulation of the pump rotational speed leads to additional energy consumption.

This study considers a hydraulic system with a curve like curve 2 in Figure 3. With this relationship between the system curve and the BEP curve, for maximum reliability, it is necessary to regulate the water flow by bypassing and throttling along with speed variation. It is assumed that in the considered parallel system centrifugal monoblock pumps CalpedaB-50/12A with a power rating of $4 \mathrm{~kW}$ and a rated rotational speed of $2900 \mathrm{rpm}$ are used. Table 1 and Figure 4 show the catalogue characteristics of this pump [27]. 
Table 1. Catalogue characteristics of centrifugal pump Calpeda-B-50/12A (4 kW, $2900 \mathrm{rpm})$.

\begin{tabular}{ccccccccc}
\hline Flow $\boldsymbol{Q}, \mathbf{~ m}^{\mathbf{3}} / \mathbf{h}$ & 30 & 33 & 37.8 & 42 & 48 & 54 & 60 & 66 \\
Head $\boldsymbol{H}, \mathbf{m}$ & 24 & 24 & 23 & 22.5 & 21 & 19.5 & 17.5 & 15 \\
Efficiency $\boldsymbol{\eta}, \%$ & 63.4 & 66.9 & 69.4 & 72.4 & 73.8 & 74.2 & 73.6 & 69.6 \\
\hline
\end{tabular}

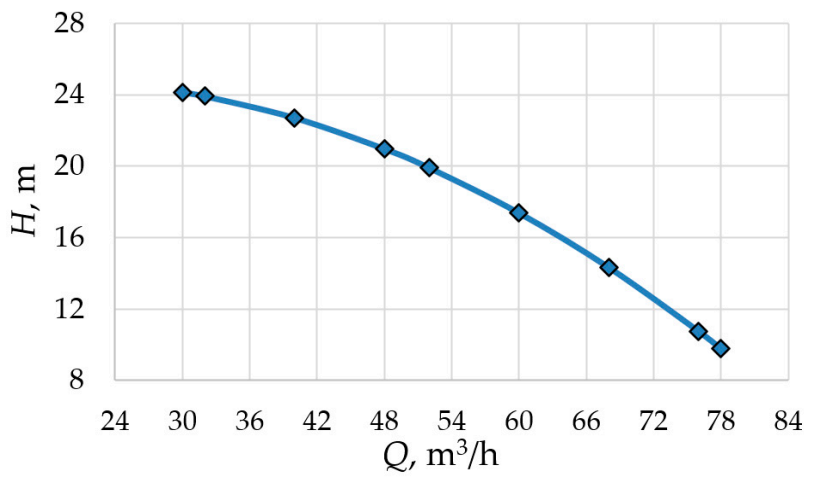

(a)

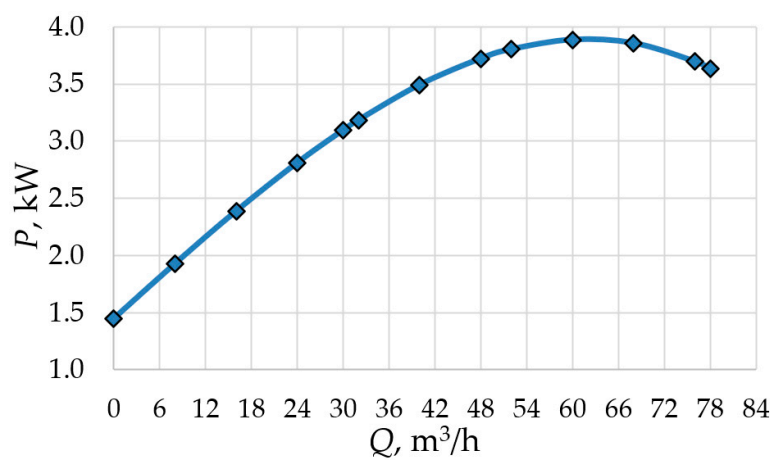

(b)

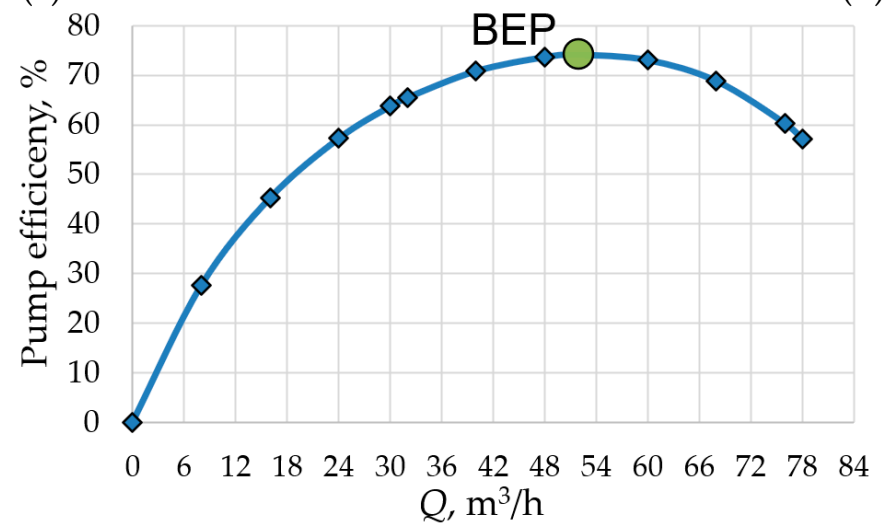

(c)

Figure 4. Data sheet characteristics for the pump Calpeda-B-50/12A versus flow rate (a) head; (b) mechanical power; (c) efficiency [27].

The maximum required flow rate of the pumping system is $Q_{\max }=120 \mathrm{~m}^{3} / \mathrm{h}$. To simplify calculations, the $Q-H$ pump characteristic and characteristic of the pump required mechanical power $P=f(Q, s)$ are interpolated as polynomials with two variables of the 2nd and 3rd order, respectively [10]:

$$
\begin{gathered}
H=a \cdot Q^{2}+b \cdot Q \cdot s+c \cdot s^{2} \\
P=c_{0} \cdot Q^{3}+c_{1} \cdot Q^{2} \cdot s+c_{2} \cdot Q \cdot s^{2}+c_{3} \cdot s^{3},
\end{gathered}
$$

where $s=n / n_{\text {rate }}$ is the relative rotational speed; $a=-0.0012, b=0.166, c=21.505$, $c_{0}=-0.0047, c_{1}=-0.053, c_{2}=60.76, c_{3}=1449$ are the coefficients of Equations (3) and (4).

Figure 5 shows the pump $H-Q$ curve at the rated speed $\left(n_{\text {rate }}\right)$, the hydraulic system curve and the BEP curve. The BEP curve is defined according to Equation (2). 


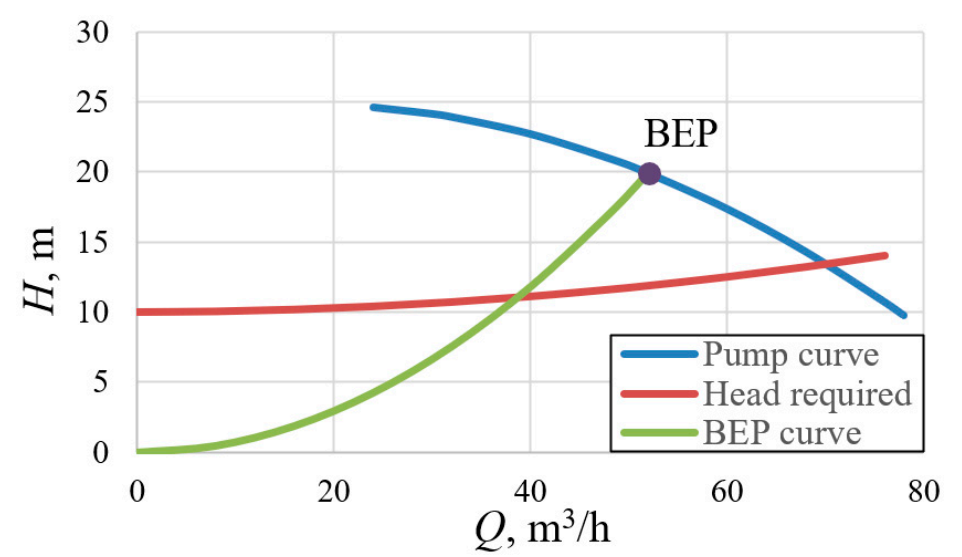

Figure 5. $Q-H$ pump characteristic, hydraulic load characteristic and BEP curve.

\section{Calculation of Characteristics of the Pumping System at Different Methods of Regulation}

This section describes the results of calculating the characteristics of the pumping system under consideration with three considered regulation methods: maximum efficiency, maximum reliability and trade-off.

Due to the wide range of variation in water flow and head in a real pumping system, the pump operating point is very rarely near the BEP. However, to ensure an acceptable service life, the pump operating point must be in the so-called preferred operating region (POR). According to [5], the POR is located between the points $0.7 \cdot Q_{B E P}$ and $1.2 \cdot Q_{B E P}$ on the $Q-H$ characteristic. This condition can be considered as a constraint when regulating the pumping system. Therefore, this paper compares the energy consumption of the pumping system in three cases:

(1) maximum efficiency (minimum power consumption) regulation, without applying any reliability constraints. In this case, the flow rate is regulated by changing only the rotational speed of the regulated (first) pump and throttling of the unregulated (second and third) pumps. When two and three pumps operate together, an equal distribution of the flow rate between them is achieved. In this case, energy consumption is minimized, but at the same time not all pump operating points are in the POR [6].

(2) maximum reliability regulation. In this case, the flow rate is regulated by changing the rotation speed of the first pump, throttling and bypass regulation of all three pumps. With this regulation, the operation of all turned-on pumps is achieved at points with maximum reliability. Due to the use of throttling and bypass, the energy consumption of the pumping system increases in comparison with the first case.

(3) trade-off regulation taking into account the POR reliability constraint. Flow rate is regulated by changing the rotation speed of the first pump, throttling and bypass the regulation of all three pumps. With this regulation, the operation of all turned-on pumps is achieved at operating points in the range from $0.7 \cdot Q_{B E P}$ to $1.2 \cdot Q_{B E P}$ in the entire required control range.

In all three considered cases, when the flow rate changes from $0 \%$ to $40 \%$, only the regulated pump runs, then the second (unregulated) pump turns on. When the flow rate reaches $80 \%$, the third (unregulated) pump also turns on.

The rest of this section describes the results of calculating the pump operating points for each of the considered regulation methods.

\subsection{Characteristics of the Pumping System with the Maximum Efficiency Regulation (Conventional Speed Regulation)}

The rotational speed of the first pump in the parallel system can be adjusted. The speeds of the second and third pumps running in parallel are not adjusted. With this control, the operating points of the variable speed pump are along the hydraulic load curve 
("head required" curve) and the operating points of the fixed speed pumps are along the catalog $\mathrm{Q}-\mathrm{H}$ curve (Figure 6).

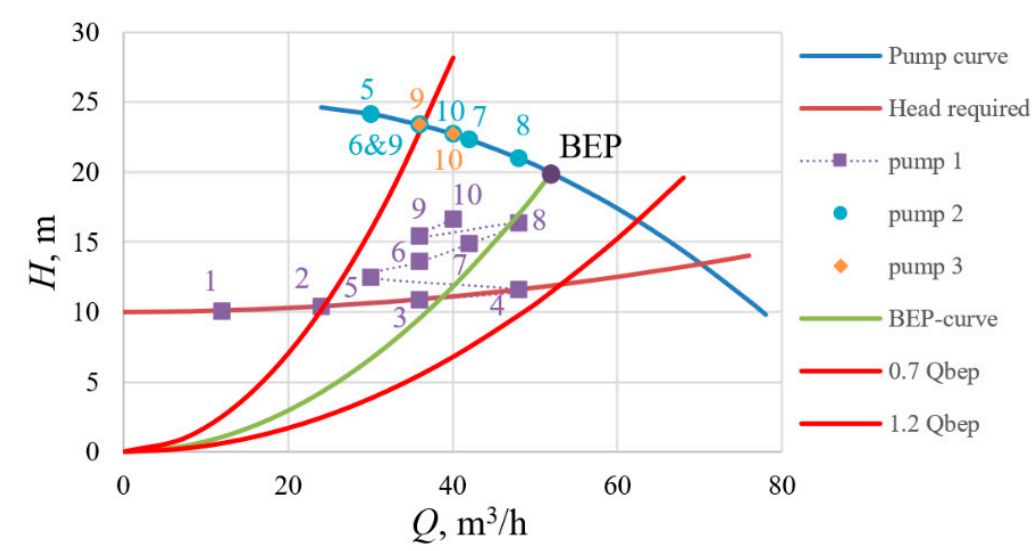

Figure 6. Operating points of the pumps when using the conventional rotational speed regulation.

The characteristics of the pumps when using this regulation method are determined as follows. The head of the variable speed pump $H_{1}=H_{R E Q}$ is determined by Equation (1), the head of the unregulated pumps is determined by Equation (3), the pump mechanical power $P$ is determined by Equation (4). The rotational speed of the variable speed pump $n$ is determined by Equation (5) [7], the pump efficiency is determined by Equation (6) and the deviation of the operating point from the BEP is determined by the Equation (7):

$$
\begin{gathered}
n_{1}=n_{\text {rate }} \cdot \frac{-b \cdot Q_{1}+\sqrt{\left(b \cdot Q_{1}\right)^{2}-4 \cdot c\left(a \cdot Q_{1}^{2}-H_{\text {req }}\right)}}{2 \cdot c} ; \\
\eta_{\mathrm{j}}=g \cdot \rho \cdot Q_{j} \cdot H_{j} / P ; \\
\theta_{\mathrm{j}}=\left(Q_{j}-Q_{B E P}\right) / Q_{B E P},
\end{gathered}
$$

where $g=9.81 \mathrm{~m} / \mathrm{s}^{2}$ is the gravitational acceleration; $\rho=1000 \mathrm{~kg} / \mathrm{m}^{3}$ is the water density; $Q_{i}$ and $H_{i}$ are the flow and the head of $j$-th pump; $\eta_{\mathrm{j}}$ and $\theta_{\mathrm{j}}$ are the efficiency and the operating poit deviation of $j$-th pump; $j=1 \ldots 3 ; Q_{B E P}=f(n)$ is the flow of the pump at $\mathrm{BEP}$ according the BEP curve Equation (2); $a, b$ and $c$ are coefficients from Equation (3).

Table 2 shows the calculation results for flow rate in the range from $10 \%$ to $100 \%$ of the maximum flow rate $Q_{\max }=120 \mathrm{~m}^{3} / \mathrm{h}$. In Table 2 and below the following characteristics of pumps 1-3 are indicated: $Q_{1} \ldots Q_{3}$ are their flow rates; $Q_{\text {req }}=Q_{1}+Q_{2}+Q_{3} ; H_{1} \ldots H_{3}$ are their hydraulic heads; $P_{1} \ldots P_{3}$ are their mechanical powers; $n_{1} \ldots n_{3}$ are their rotational speeds; $P \Sigma=P_{1}+P_{2}+P_{3} ; \eta_{1}-\eta_{3}$ are their efficiencies; $\theta_{1} \ldots \theta_{3}$ are the deviations of the operating point of the pumps. From Figure 6 and Table 2, it can be seen that the estimated deviations $\theta$ exceed $30.8 \%$ and reach $63.9 \%$, which are outside the POR in Table 2.

Such points with the large deviations occur at all stages of the flow regulation: when the required water flow is less than $20 \%$ of the $Q_{\max }$ (when only the first pump is running), at the flow rate in the range of $50-80 \%$ (the second pump turns on), and also when the flow rate is more than $80 \%$ (the third pump turns on). If the flow rate is below $0.7 \cdot Q_{B E P}$ pump operation may become unstable due to the flatness of the $Q-H$ curve: a small deviation in the rotational speed leads to a large deviation in the flowrate. In addition, due to the unstable shape of the $Q-H$ characteristic, the latter intersects the hydraulic system curve in two points which leads to the occurrence of a surge. In this case, the pump operates alternately in different operating points, the whole system is unstable, the loading on the pump changes and hydraulic shocks occur [28,29]. 
Table 2. The characteristics of the parallel pumping system using the conventional speed control.

\begin{tabular}{|c|c|c|c|c|c|c|c|c|c|c|c|c|c|c|c|}
\hline $\begin{array}{c}\text { Point } \\
\text { Number } i\end{array}$ & $\underset{\%}{Q_{r e q}}$ & $\begin{array}{l}Q_{r e q}, \\
\mathrm{~m}^{3} / \mathrm{h}\end{array}$ & $\begin{array}{c}Q_{1} \\
\mathrm{~m}^{3} / \mathrm{h}\end{array}$ & $\begin{array}{c}Q_{2} \\
\mathrm{~m}^{3} / \mathrm{h}\end{array}$ & $\begin{array}{c}Q_{3} \\
\mathrm{~m}^{3} / \mathrm{h}\end{array}$ & $\begin{array}{c}H_{1}=H_{r e q} \\
\mathrm{~m}\end{array}$ & $\begin{array}{c}\mathrm{H}_{2} \\
\mathrm{~m}\end{array}$ & $\begin{array}{c}\mathrm{H}_{3} \\
\mathrm{~m}\end{array}$ & $\begin{array}{r}n_{1} \\
\text { rpm }\end{array}$ & $\begin{array}{c}n_{2} \\
\text { rpm }\end{array}$ & $\begin{array}{c}n_{3}, \\
\text { rpm }\end{array}$ & $\begin{array}{l}P_{1} \\
\text { kW }\end{array}$ & $\begin{array}{l}P_{2} \\
\mathrm{~kW}\end{array}$ & $\begin{array}{l}P_{3} \\
\mathrm{~kW}\end{array}$ & 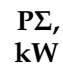 \\
\hline 1 & 10 & 12 & 12 & - & - & 10.1 & - & - & 1851 & - & - & 0.66 & - & - & 0.66 \\
\hline 2 & 20 & 24 & 24 & - & - & 10.4 & - & - & 1934 & - & - & 0.99 & - & - & 0.99 \\
\hline 3 & 30 & 36 & 36 & - & - & 10.9 & - & - & 2110 & - & - & 1.45 & - & - & 1.45 \\
\hline 4 & 40 & 48 & 48 & - & - & 11.6 & - & - & 2351 & - & - & 2.07 & - & - & 2.07 \\
\hline 5 & 50 & 60 & 30 & 30 & - & 12.5 & 24.1 & - & 2155 & 2900 & - & 1.44 & 3.09 & - & 4.53 \\
\hline 6 & 60 & 72 & 36 & 36 & - & 13.6 & 23.4 & - & 2301 & 2900 & - & 1.83 & 3.35 & - & 5.17 \\
\hline 7 & 70 & 84 & 42 & 42 & - & 14.9 & 22.3 & - & 2466 & 2900 & - & 2.31 & 3.56 & - & 5.87 \\
\hline 8 & 80 & 96 & 48 & 48 & - & 16.4 & 21.0 & - & 2644 & 2900 & - & 2.89 & 3.72 & - & 6.61 \\
\hline 9 & 90 & 108 & 36 & 36 & 36 & 15.4 & 23.4 & 23.4 & 2421 & 2900 & 2900 & 2.09 & 3.35 & 3.35 & 8.79 \\
\hline 10 & 100 & 120 & 40 & 40 & 40 & 16.7 & 22.7 & 22.7 & 2549 & 2900 & 2900 & 2.48 & 3.49 & 3.49 & 9.47 \\
\hline \multicolumn{2}{|c|}{ Point number $i$} & \multicolumn{2}{|c|}{$\eta_{1}, \%$} & \multicolumn{2}{|c|}{$\eta_{2}, \%$} & \multicolumn{2}{|l|}{$\eta_{3}, \%$} & \multicolumn{3}{|c|}{$\theta_{1}, \%$} & \multicolumn{3}{|c|}{$\theta_{2}, \%$} & \multicolumn{2}{|c|}{$\theta_{3}, \%$} \\
\hline \multicolumn{2}{|l|}{1} & \multicolumn{2}{|c|}{50.0} & \multicolumn{2}{|c|}{-} & \multicolumn{2}{|c|}{-} & \multicolumn{3}{|c|}{-63.9} & \multicolumn{3}{|c|}{-} & \multicolumn{2}{|c|}{-} \\
\hline \multicolumn{2}{|l|}{2} & \multicolumn{2}{|c|}{68.5} & \multicolumn{2}{|c|}{-} & \multicolumn{2}{|l|}{-} & \multicolumn{3}{|c|}{-30.8} & \multicolumn{3}{|c|}{-} & \multicolumn{2}{|c|}{-} \\
\hline \multicolumn{2}{|l|}{3} & \multicolumn{2}{|c|}{73.9} & \multicolumn{2}{|c|}{-} & \multicolumn{2}{|l|}{-} & \multicolumn{3}{|c|}{-4.8} & \multicolumn{3}{|c|}{-} & \multicolumn{2}{|c|}{-} \\
\hline \multicolumn{2}{|l|}{4} & \multicolumn{2}{|c|}{73.3} & & & - & & & 13.9 & & & - & & & \\
\hline 5 & & & & & & - & & & -22.4 & & & -42.3 & & & \\
\hline 6 & & & & & & - & & & -12.8 & & & -30.8 & & & \\
\hline 7 & & & & & & - & & & -5.0 & & & -19.2 & & & \\
\hline 8 & & & & & & - & & & 1.2 & & & -7.7 & & & \\
\hline 9 & & & & & & 68.5 & & & -17.1 & & & -30.8 & & & \\
\hline 10 & & & & & & 70.9 & & & -12.5 & & & -23.1 & & & 3.1 \\
\hline
\end{tabular}

\subsection{Characteristics of the Pumping System with the Maximum Reliability Regulation}

For maximum pump reliability, the operating points of the variable speed pump must be on the BEP curve (dashed line in Figure 3), and fixed-speed pumps must always run at the BEP point on the catalog pump $Q-H$ curve, regardless of the required flow rate. This can only be achieved by using all three of the above-mentioned water flow regulation methods together.

As shown in Figure 6, variable speed pump operating points 1-3, 5-7 and 9-10 are located to the left of the BEP curve. To implement the condition of maximum reliability, these points are to move horizontally until they coincide with the BEP curve. All operating points of the fixed-speed pumps are also located to the left of the BEP curve. They need to be moved along the catalog $Q-H$ curve up to the BEP. This means that the pump flow rate $Q_{i}$ will increase from $Q_{i}{ }^{\prime}$ to $Q_{B E P}$ at the same head. Excess flow $Q_{i}-Q_{i}{ }^{\prime}$ flows back through the bypass to the suction pipe, where. In this case $Q_{r e q}=Q_{1^{\prime}}+Q_{2^{\prime}}+Q_{3^{\prime}}$.

Points 4 and 5 are located to the right of the BEP curve. To move these points up to the BEP curve, at a constant flow rate $Q_{\text {req }}$, the head at the pump outlet must be increased by throttling (Figure 7).

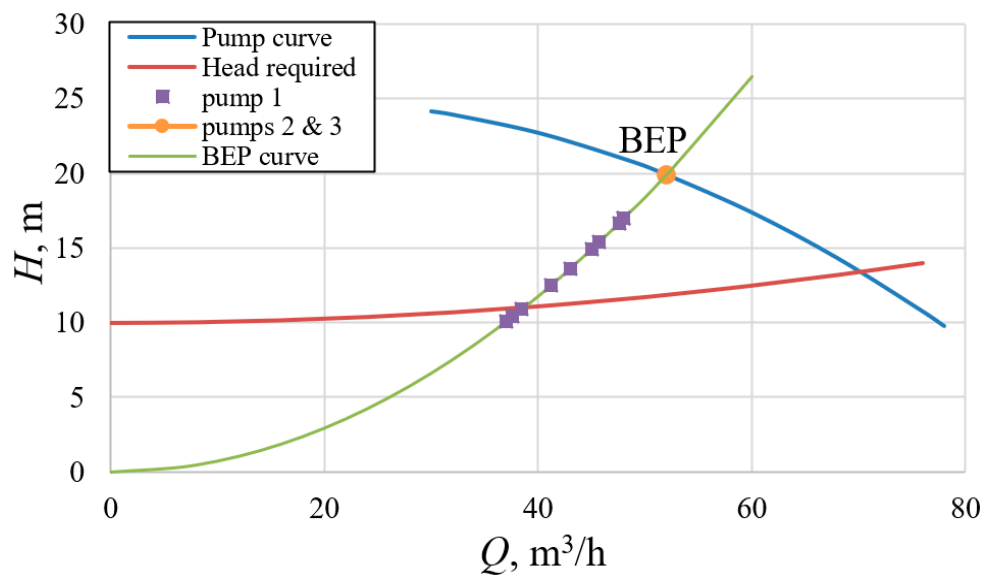

Figure 7. Operating points of the pump when using the maximum reliability regulation. 
The required head $H_{\text {req }}$ is determined by the Equation (1). $H_{\text {req }}$ is the same for all switched on pumps as they operate in parallel. The flow $\mathrm{Q}_{j}$ and the head $H_{j}$ are determined by Equation (8) when regulating the bypass together with speed regulation or by Equation (9) when regulating only the pump rotational speed. The rest of the parameters are determined in the same way as in the previous section:

$$
\begin{gathered}
Q_{j}=\sqrt{ }\left(\mathrm{H}_{\text {req }} / \mathrm{k}_{\mathrm{BEP}}\right) ; \mathrm{H}_{j}=\mathrm{H}_{\text {req }}, \\
\mathrm{Q}_{j}=\mathrm{Q}_{\text {req }} ; \mathrm{H}_{j}=\mathrm{k}_{\mathrm{BEP}} \cdot \mathrm{Q}_{\mathrm{req}}{ }^{2},
\end{gathered}
$$

where $Q_{r e q}$ and $H_{r e q}$ are the required output flow and head; $\mathrm{Q}_{j}$ and $\mathrm{H}_{j}$ are the flow and head of $j$-th pump.

Table 3 shows the calculated characteristics of the pump system with the maximum reliability regulation. As the results in Figure 7 in Table 3 show, when the throttling and bypass are used in combination with the speed control, the maximum pump reliability can be achieved. Operating point deviation $\theta$ is zero.

\begin{tabular}{|c|c|c|c|c|c|c|c|c|c|c|c|c|c|c|}
\hline \multirow[b]{2}{*}{$\begin{array}{c}\text { Point } \\
\text { Number } i\end{array}$} & \multirow[b]{2}{*}{$\begin{array}{l}Q_{\text {req }} \\
\mathrm{m}^{3} / \mathrm{h}\end{array}$} & \multirow[b]{2}{*}{$\begin{array}{c}H_{r e q} \\
\text { m }\end{array}$} & \multicolumn{4}{|c|}{ Pump $1(j=1)$} & \multicolumn{4}{|c|}{ Pump $2(j=2)$} & \multicolumn{4}{|c|}{ Pump $3(j=3)$} \\
\hline & & & $\begin{array}{c}Q_{1^{\prime}} \\
\mathbf{m}^{3} / \mathbf{h}\end{array}$ & $\underset{\mathrm{m}^{3} / \mathrm{h}}{Q_{1}}$ & $\begin{array}{c}H_{1} \\
\text { m }\end{array}$ & $\begin{array}{c}n_{1} \\
\text { rpm }\end{array}$ & $\begin{array}{c}Q_{2^{\prime}} \\
\mathrm{m}^{3} / \mathbf{h}\end{array}$ & $\begin{array}{c}Q_{2} \\
\mathbf{m}^{3} / \mathbf{h}\end{array}$ & $\begin{array}{c}H_{2} \\
\text { m }\end{array}$ & $\begin{array}{c}n_{2} \\
\text { rpm }\end{array}$ & $\begin{array}{c}Q_{3^{\prime}} \\
\mathrm{m}^{3} / \mathbf{h}\end{array}$ & $\begin{array}{c}Q_{3} \\
\mathrm{~m}^{3} / \mathrm{h}\end{array}$ & $\begin{array}{c}H_{3} \\
\mathbf{m}\end{array}$ & $\begin{array}{r}n_{3} \\
\text { rpm }\end{array}$ \\
\hline 1 & 12 & 10.1 & 12 & 37.0 & 10.1 & 2066 & - & - & - & - & - & - & - & - \\
\hline 2 & 24 & 10.4 & 24 & 37.6 & 10.4 & 2096 & - & - & - & - & - & - & - & - \\
\hline 3 & 36 & 10.9 & 36 & 38.5 & 10.9 & 2146 & - & - & - & - & - & - & - & - \\
\hline 4 & 48 & 11.6 & 48 & 48.0 & 16.9 & 2677 & - & - & - & - & - & - & - & - \\
\hline 5 & 60 & 12.5 & 30 & 41.2 & 12.5 & 2298 & 30 & 52.0 & 19.9 & 2900 & - & - & - & - \\
\hline 6 & 72 & 13.6 & 36 & 43.0 & 13.6 & 2397 & 36 & 52.0 & 19.9 & 2900 & - & - & - & - \\
\hline 7 & 84 & 14.9 & 42 & 45.0 & 14.9 & 2509 & 42 & 52.0 & 19.9 & 2900 & - & - & - & - \\
\hline 8 & 96 & 16.4 & 48 & 48.0 & 16.9 & 2677 & 48 & 52.0 & 19.9 & 2900 & - & - & - & - \\
\hline 9 & 108 & 15.4 & 36 & 45.7 & 15.4 & 2551 & 36 & 52.0 & 19.9 & 2900 & 36 & 52.0 & 19.9 & 2900 \\
\hline 10 & 120 & 16.7 & 40 & 47.6 & 16.7 & 2654 & 40 & 52.0 & 19.9 & 2900 & 40 & 52.0 & 19.9 & 2900 \\
\hline \multicolumn{2}{|c|}{ Point number $i$} & \multicolumn{2}{|c|}{$P_{1}, \mathrm{~kW}$} & \multicolumn{2}{|c|}{$P_{2}, \mathrm{~kW}$} & \multicolumn{2}{|c|}{$P_{3}, \mathrm{~kW}$} & $\mathrm{P} \Sigma, \mathrm{W}$ & $\eta_{1}, \%$ & $\eta_{2}, \%$ & $\eta_{3}, \%$ & $\theta_{1}, \%$ & $\theta_{2}, \%$ & $\theta_{3}, \%$ \\
\hline \multicolumn{2}{|c|}{1} & \multicolumn{2}{|c|}{1.37} & \multicolumn{2}{|c|}{-} & \multicolumn{2}{|c|}{-} & 1.37 & 74.2 & - & - & 0 & - & - \\
\hline \multicolumn{2}{|l|}{2} & \multicolumn{2}{|c|}{1.44} & \multicolumn{2}{|c|}{-} & \multicolumn{2}{|c|}{-} & 1.44 & 74.2 & - & - & 0 & - & - \\
\hline \multicolumn{2}{|l|}{3} & \multicolumn{2}{|c|}{1.54} & \multicolumn{2}{|c|}{-} & \multicolumn{2}{|c|}{-} & 1.54 & 74.2 & - & - & 0 & - & - \\
\hline \multicolumn{2}{|l|}{4} & \multicolumn{2}{|c|}{2.99} & \multicolumn{2}{|c|}{-} & \multicolumn{2}{|c|}{-} & 2.99 & 74.2 & - & - & 0 & - & - \\
\hline \multicolumn{2}{|l|}{5} & \multicolumn{2}{|c|}{1.89} & \multicolumn{2}{|c|}{3.80} & \multicolumn{2}{|c|}{-} & 5.70 & 74.2 & 74.2 & - & 0 & 0 & - \\
\hline 6 & & & & & & & - & 5.95 & 74.2 & 74.2 & - & 0 & 0 & - \\
\hline 7 & & & & & & & - & 6.27 & 74.2 & 74.2 & - & 0 & 0 & - \\
\hline 8 & & & & & & & - & 6.79 & 74.2 & 74.2 & - & 0 & 0 & - \\
\hline 9 & & & & & & & 80 & 10.2 & 74.2 & 74.2 & 74.2 & 0 & 0 & 0 \\
\hline 10 & & & & & & & 80 & 10.5 & 74.2 & 74.2 & 74.2 & 0 & 0 & 0 \\
\hline
\end{tabular}

Table 3. The characteristics of the parallel pumping system using the maximum reliability regulation.

The pump efficiency throughout the entire flow regulation range remains at its maximum (74.2\%). However, power consumption increases compared to the conventional speed regulation due to the increase in $Q_{P U M P}$ when using the bypass and the increase in $H_{\text {PUMP }}$ when using the throttling.

\subsection{Characteristics of the Pumping System with the Trade-Off Regulation}

It is also possible to apply a trade-off regulation method to reduce energy consumption and, at the same time, reach sufficiently high reliability. To achieve this, it is necessary to ensure that all pump operating points are located within the POR. According to Figure 6, when using only the speed control, points 1,2 of the first regulated pump, points 5, 6 of the second pump and point 9 of the third pump are outside of the POR.

In this case, as in the case of the maximum reliability regulation, points 1, 2 of the first pump are moved horizontally but only to the right to the border of the POR (1.2. $Q_{B E P}$ line) using a bypass (Figure 8). Furthermore, points 5, 6 of the second pump and point 9 of the third pump move along the $Q-H$-curve to the left border of the POR (0.7- $Q_{B E P}$ line). Thus, all operating points are restricted by the POR. As in the previous case, $H_{r e q}$ is the 
same for all switched on pumps as they operate in parallel and $Q_{r e q}=Q_{1^{\prime}}+Q_{2^{\prime}}+Q_{3^{\prime}}$. This ensures high reliability and low power consumption. Throttling is not applied in this case. The characteristics of pumps with the trade-off regulation are determined as follows. The required head of the variable speed pump $H_{\text {req }}$ is determined using Equation (1).

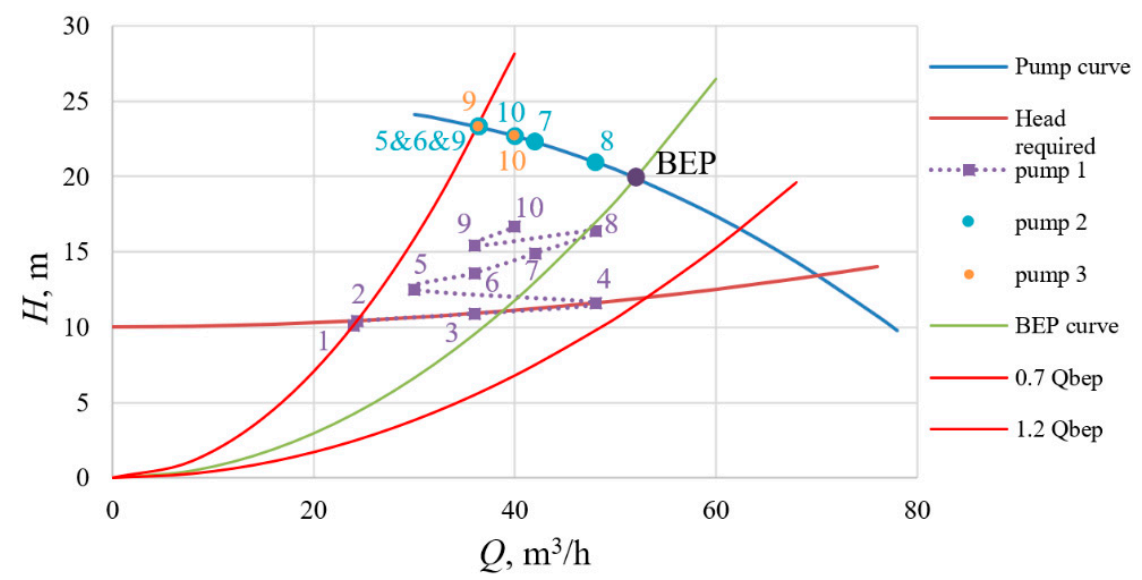

Figure 8. Operating points of the pumps when using the trade-off regulation.

The flow through the pump $Q_{j}$ and the head $H_{j}$ are determined by Equation (10) with bypass regulation. The rest of the parameters are determined in the same way as in the case of the conventional speed regulation:

$$
Q_{j}=\sqrt{ }\left(H_{R E Q} / k_{0.7 B E P}\right) ; H_{j}=H_{R E Q},
$$

where $k_{0.7 B E P}=H\left(0.7 \cdot Q_{B E P}\right) /\left(0.7 \cdot Q_{B E P}\right)$.

Table 4 and Figure 8 show the calculated characteristics of the pump system applying the proposed trade-off regulation. As the results in Table 4 show, with the proposed tradeoff regulation method, the efficiency of the pumps in the entire range of the flow control is not less than $68.8 \%$. The flow deviation $\theta$ of the operating points from the BEP is no more than $30 \%$, and the energy consumption is reduced compared to the maximum reliability regulation method.

Table 4. The characteristics of the parallel pumping system using the trade-off regulation.

\begin{tabular}{|c|c|c|c|c|c|c|c|c|c|c|c|c|c|c|}
\hline \multirow[b]{2}{*}{$\begin{array}{c}\text { Point } \\
\text { Number } i\end{array}$} & \multirow[b]{2}{*}{$\begin{array}{c}Q_{r e q} \\
\mathrm{~m}^{3} / \mathrm{h}\end{array}$} & \multirow[b]{2}{*}{$H_{r e q}, \mathrm{~m}$} & \multicolumn{4}{|c|}{ Pump $1(j=1)$} & \multicolumn{4}{|c|}{ Pump $2(j=2)$} & \multicolumn{4}{|c|}{ Pump $3(j=3)$} \\
\hline & & & $\begin{array}{c}Q_{1^{\prime}} \\
\mathbf{m}^{3} / \mathbf{h}\end{array}$ & $\underset{\mathbf{m}^{3} / \mathbf{h}}{Q_{1}}$ & $H_{1}, \mathrm{~m}$ & $\begin{array}{r}n_{1} \\
\text { rpm }\end{array}$ & $\begin{array}{c}Q_{2^{\prime}} \\
\mathrm{m}^{3} / \mathrm{h}\end{array}$ & $\begin{array}{c}Q_{2} \\
\mathbf{m}^{3} / \mathbf{h}\end{array}$ & $\begin{array}{c}\mathrm{H}_{2} \\
\mathrm{~m}\end{array}$ & $\begin{array}{r}n_{2} \\
\text { rpm }\end{array}$ & $\begin{array}{c}Q_{3^{\prime}} \\
\mathrm{m}^{3} / \mathbf{h}\end{array}$ & $\begin{array}{r}Q_{3} \\
\mathbf{m}^{3} / \mathbf{h}\end{array}$ & $H_{3}, \mathrm{~m}$ & $\begin{array}{c}n_{3} \\
\text { rpm }\end{array}$ \\
\hline 1 & 12 & 10.1 & 12 & 24.0 & 10.1 & 1909 & - & - & - & - & - & - & - & - \\
\hline 2 & 24 & 10.4 & 24 & 24.3 & 10.4 & 1937 & - & - & - & - & - & - & - & - \\
\hline 3 & 36 & 10.9 & 36 & 36.0 & 10.9 & 2110 & - & - & - & - & - & - & - & - \\
\hline 4 & 48 & 11.6 & 48 & 48.0 & 11.6 & 2351 & - & - & - & - & - & - & - & - \\
\hline 5 & 60 & 12.5 & 30 & 30.0 & 12.5 & 2155 & 30 & 36.4 & 23.3 & 2900 & - & - & - & - \\
\hline 6 & 72 & 13.6 & 36 & 36.0 & 13.6 & 2301 & 36 & 36.4 & 23.3 & 2900 & - & - & - & - \\
\hline 7 & 84 & 14.9 & 42 & 42.0 & 14.9 & 2466 & 42 & 42.0 & 22.3 & 2900 & - & - & - & - \\
\hline 8 & 96 & 16.4 & 48 & 48.0 & 16.4 & 2644 & 48 & 48.0 & 21.0 & 2900 & - & - & - & - \\
\hline 9 & 108 & 15.4 & 36 & 36.0 & 15.4 & 2421 & 36 & 36.4 & 23.3 & 2900 & 36 & 36.4 & 23.3 & 2900 \\
\hline 10 & 120 & 16.7 & 40 & 40.0 & 16.7 & 2549 & 40 & 40.0 & 22.7 & 2900 & 40 & 40.0 & 22.7 & 2900 \\
\hline \multicolumn{2}{|c|}{ Point number $i$} & $P_{1}, \mathrm{~kW}$ & \multicolumn{2}{|c|}{$P_{2}, \mathrm{~kW}$} & $P_{3}, \mathrm{~kW}$ & \multirow{2}{*}{\multicolumn{2}{|c|}{$\begin{array}{c}\mathbf{P \Sigma}, \mathbf{W} \\
0.96\end{array}$}} & $\eta_{1}, \%$ & \multicolumn{2}{|c|}{$\eta_{2}, \%$} & $\eta_{3}, \%$ & $\theta_{1}, \%$ & $\theta_{2}, \%$ & $\theta_{3}, \%$ \\
\hline \multirow{2}{*}{\multicolumn{2}{|c|}{$\begin{array}{l}1 \\
2\end{array}$}} & 0.96 & \multicolumn{2}{|c|}{-} & - & & & 68.8 & \multicolumn{2}{|c|}{-} & - & -30.0 & - & - \\
\hline & & 1.00 & \multicolumn{2}{|c|}{-} & - & \multicolumn{2}{|c|}{1.00} & 68.8 & \multicolumn{2}{|c|}{-} & - & -30.0 & - & - \\
\hline \multicolumn{2}{|c|}{3} & 1.45 & \multicolumn{2}{|c|}{-} & - & \multicolumn{2}{|c|}{1.45} & 73.9 & \multicolumn{2}{|c|}{-} & - & -4.8 & - & - \\
\hline \multicolumn{2}{|c|}{4} & 2.07 & \multicolumn{2}{|c|}{-} & - & \multicolumn{2}{|c|}{2.07} & 73.3 & \multicolumn{2}{|c|}{ - } & - & 13.9 & - & - \\
\hline \multicolumn{2}{|c|}{5} & 1.44 & \multicolumn{2}{|c|}{3.36} & - & \multicolumn{2}{|c|}{4.80} & 71.1 & \multicolumn{2}{|c|}{68.8} & - & -22.4 & -30.0 & - \\
\hline \multicolumn{2}{|c|}{6} & 1.83 & \multicolumn{2}{|c|}{3.36} & - & & & 73.0 & & & - & -12.8 & -30.0 & - \\
\hline 7 & & 2.31 & & & - & & & 73.9 & & & - & -5.0 & -19.2 & - \\
\hline 8 & & 2.89 & & & - & & & 74.2 & & & - & 1.2 & -7.7 & - \\
\hline 9 & & 2.09 & & & 3.36 & & & 72.3 & & & 68.8 & -17.1 & -30.0 & -30.0 \\
\hline 10 & & 2.49 & & & 3.49 & & & 73.1 & & & 70.9 & -12.5 & -23.1 & -23.1 \\
\hline
\end{tabular}




\section{Comparison of Energy Consumption and Reliability of the Parallel System with Different Regulation Methods}

Figures 9-12 summarize the results of Tables 2-4. Figure 9 compares the flow rates of individual pumps with different control methods. With the maximum efficiency method, the flow of each pump is minimized, but the deviations of $\theta$ at some points are below the POR limit. When applying maximum reliability regulation, the pump flow is always increased by applying a bypass to keep all pumps running at the BEP. When applying the trade-off regulation, if $\theta<-30 \%$, then the pump flow increases so that $\theta=-30 \%$.

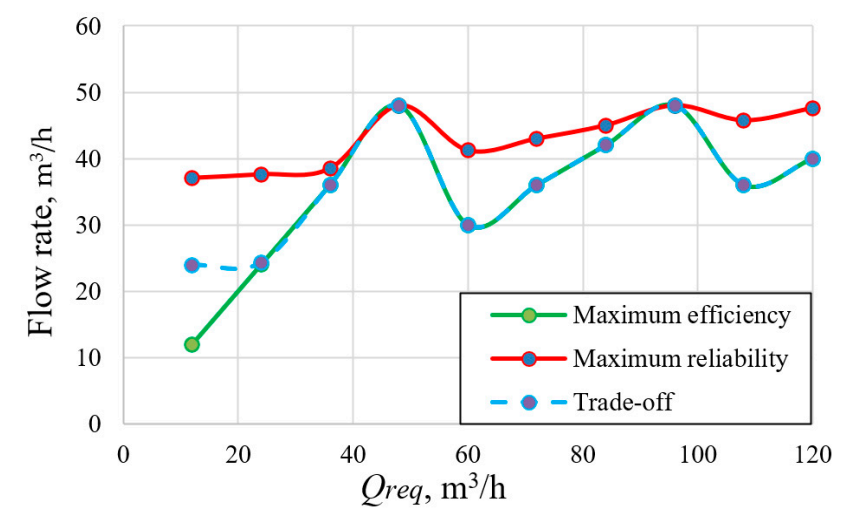

(a)

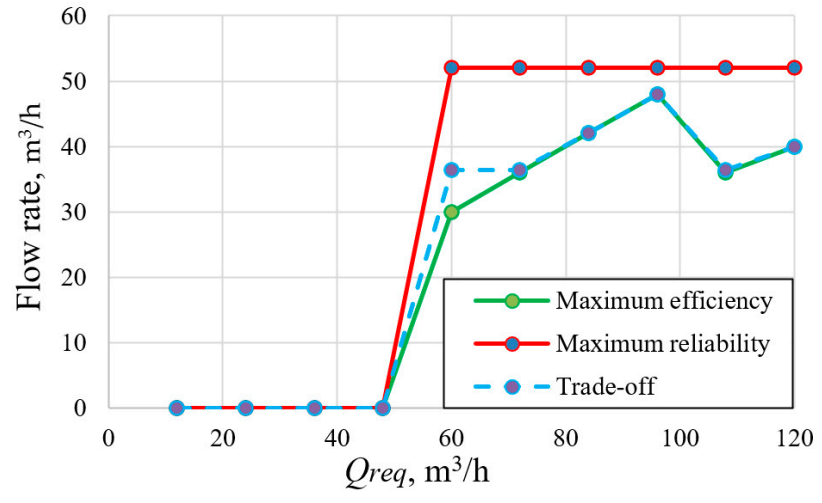

(b)

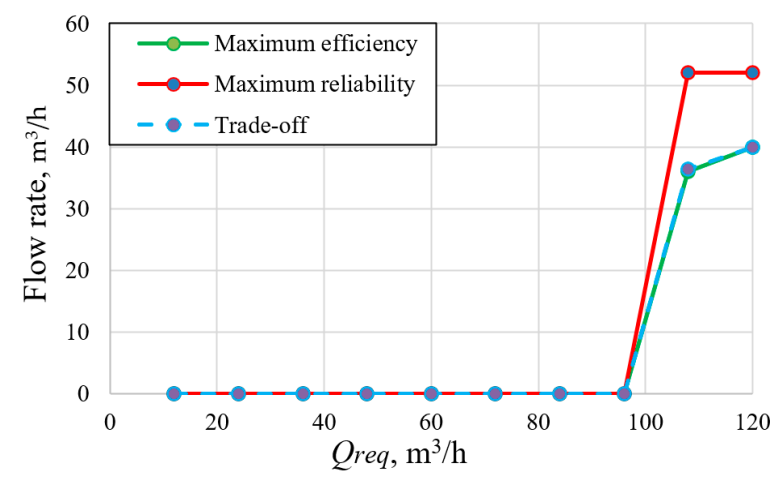

(c)

Figure 9. Comparison of flow rates of individual pumps with different regulation methods (a) pump $1(j=1)$; (b) pump 2 $(j=2)$; (c) pump $3(j=3)$.

Figure 10 compares the efficiency of individual pumps with different control methods. With the maximum reliability method, the efficiency of all pumps is always maximized since they always run at the BEP. However, the total energy consumption of the pumping system in this case is also maximum (Figure 11) since the additional energy consumption from throttling and bypass exceeds the benefit from improving the efficiency of the pumps.

Figures 11 and 12 compare the required mechanical power and the pump operating point deviation from BEP $\theta$ at the various regulation methods considered. Figure 11 shows that when using the maximum reliability regulation, the required mechanical power significantly increases compared to the conventional speed regulation due to the use of bypass and throttling. At the same time, when using the trade-off regulation, the required mechanical power only slightly increases at two operating points.

According to the proposed trade-off regulation principle, it is necessary to correct only the operating points which deviations $\theta$ are outside the POR boundaries (marked in red in Table 2). As Figure 6 and Table 2 show, most of the operating points of the three pumps are already within the POR when the "maximum efficiency" control is applied. Therefore, when the trade-of regulation is applied, most of the operating points remain 
unchanged, in comparison with the maximum efficiency regulation method. In addition, 4 of 6 points with an unacceptable deviation $\theta$ have a deviation value of $30.8 \%$, which is very close to the permissible limit value of $30 \%$. Therefore, the correction of these points when using the trade-off regulation does not require significant additional energy consumption. Significant deviations $\theta$ only need to be corrected for the first pump $(j=1)$ at point $1(i=1)$ and for the second pump $(j=1)$ at point $5(i=5)$. This results in a very similar shape of the graphs for $P \Sigma$ and $\theta$ in Figures 11 and 12. Only for the correction of operating points 1 and 5 significant additional power consumption is required, which is reflected in Figure 11d. Figure 12 shows that the proposed trade-off regulation maintains pump duty points within POR over the entire required flow range.

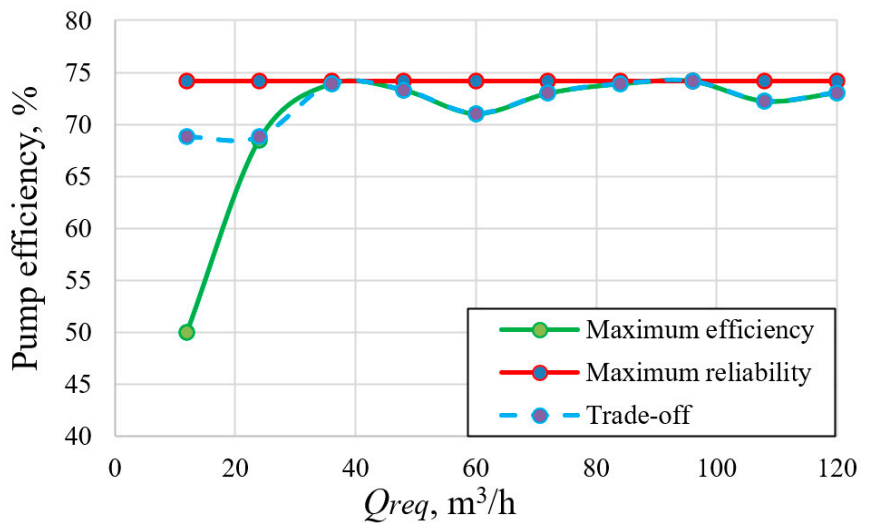

(a)

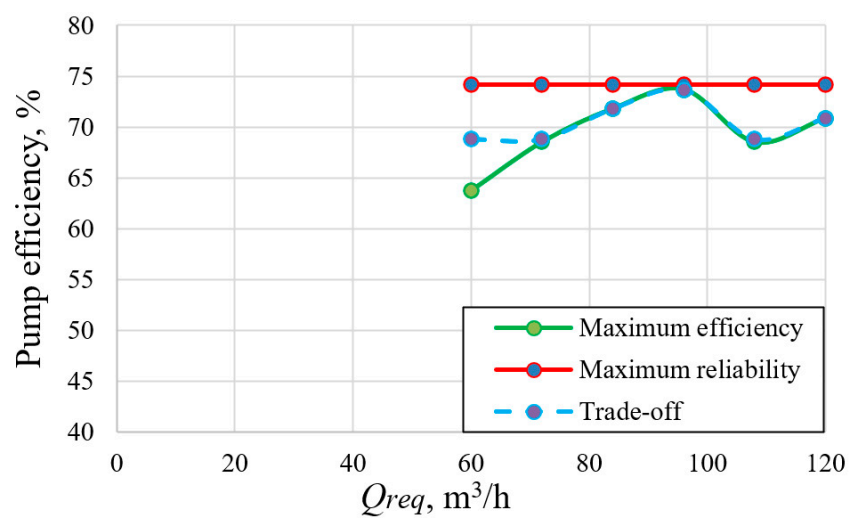

(b)

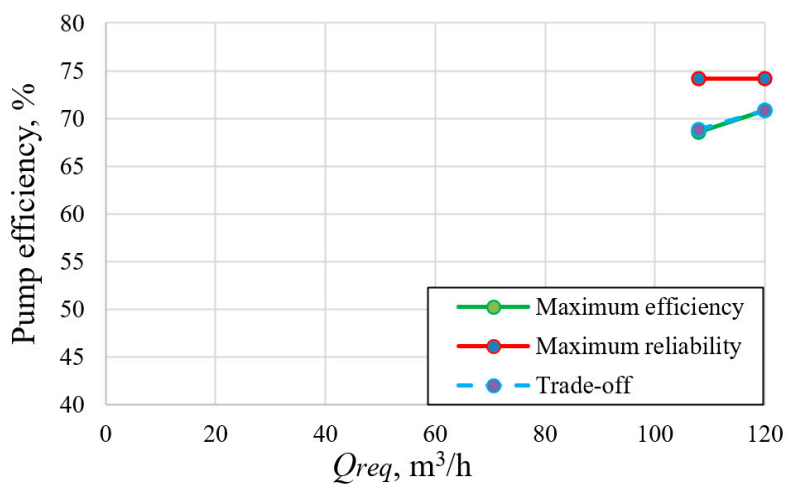

(c)

Figure 10. Comparison of efficiencies of individual pumps with different regulation methods (a) pump 1 ( $j=1)$; (b) pump 2 $(j=2)$; (c) pump $3(j=3)$.

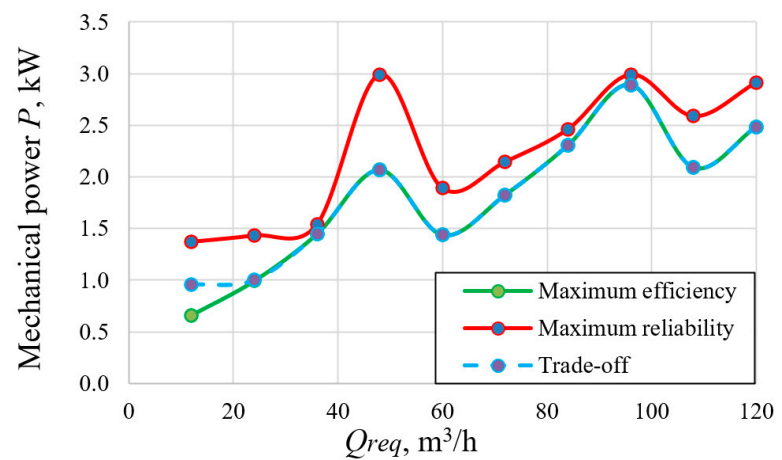

(a)

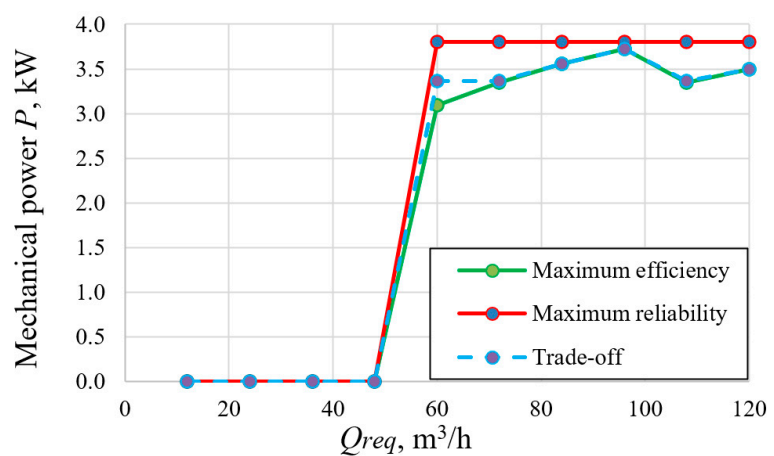

(b)

Figure 11. Cont. 


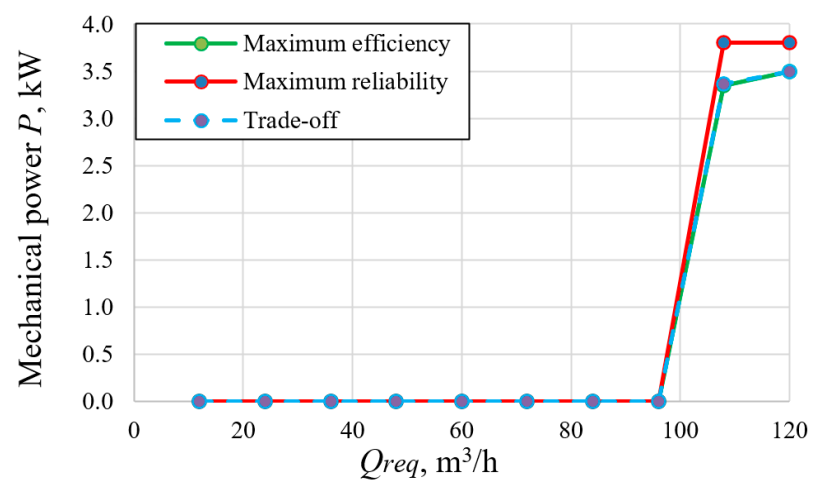

(c)

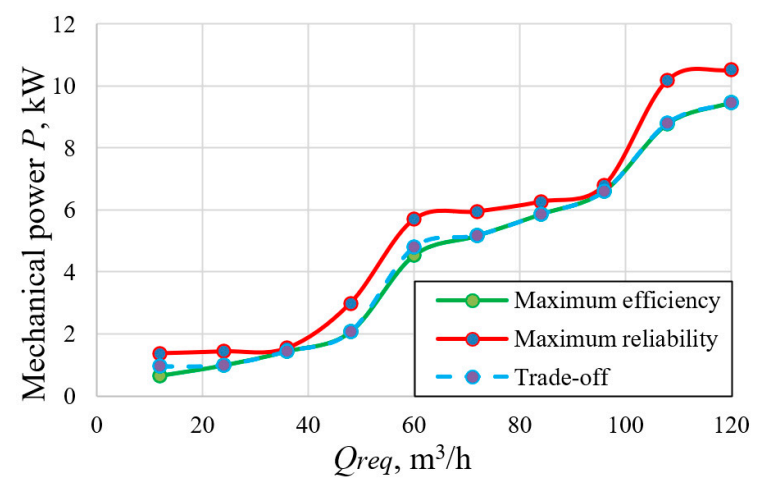

(d)

Figure 11. Comparison of the mechanical power of the pumps with different regulation methods (a) pump $1\left(P_{1}\right)$; (b) pump $2\left(P_{2}\right)$; (c) pump $3\left(P_{3}\right)$; (d) sum of mechanical power $(P \Sigma)$.

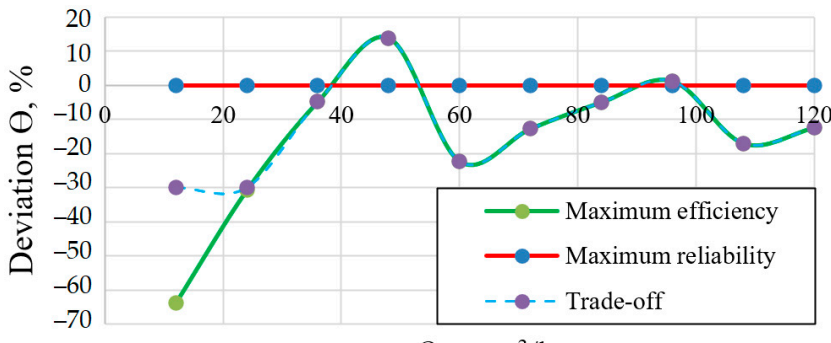

Qreq, $\mathrm{m}^{3} / \mathrm{h}$

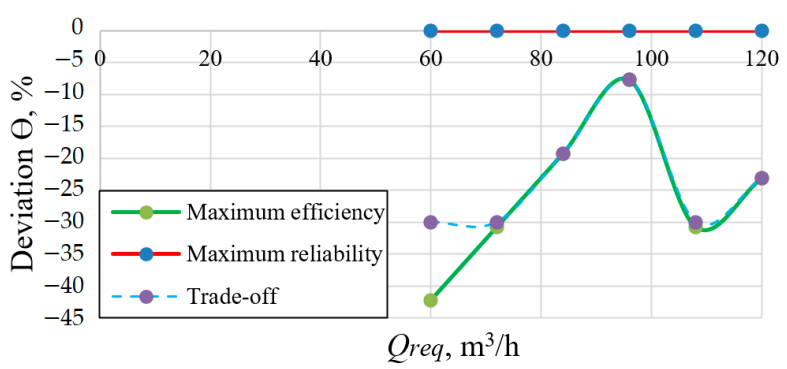

(b) (a)

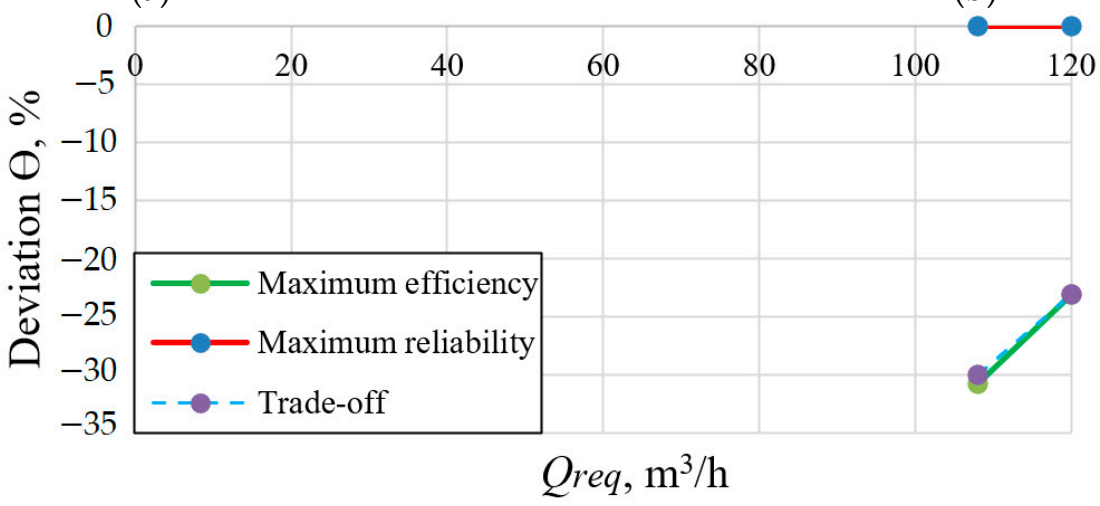

(c)

Figure 12. Comparison of deviations $\theta$ of individual pumps with different regulation methods (a) pump $1(j=1)$; (b) pump $2(j=2)$; (c) pump $3(j=3)$.

Based on the obtained results (Figure 11), the energy consumption of the parallel pumping system is compared when operating with a duty cycle corresponding to a typical open-type pumping system [26]. Figure 13 shows the diagram of the duty cycle. The duty cycle period is $24 \mathrm{~h}$. Electricity consumption is compared at three regulation methods under consideration.

When calculating the electrical power consumption of a pump unit, it is also necessary to consider the characteristics of the components of its electric drive. It was assumed that Sinamics G120C frequency converter with the rated power of $4 \mathrm{~kW}$ [30] and Simotics 1LE1001-1CA6 induction motors with the power of $4 \mathrm{~kW}$ and rated speed of $2955 \mathrm{rpm}$ [31] are used in the drive of the pump system. Data from SinaSave software [32] in 8 standard loading points are used to determine the power losses and efficiency of the drive (motor plus frequency converter) in the operating points with given values of the shaft torque 
$T$ and speed $n$ that were calculated in the previous section (Tables 2-4). The standard loading points are determined according to IEC 61800-9-2, "Adjustable Speed Electrical Power Drive Systems-Part 9-2: Ecodesign for Power Drive Systems, Motor Starters, Power Electronics and Their Driven Applications-Energy Efficiency Indicators for Power Drive Systems and Motor Starters" [33]. These data are used because the standard [33] requires manufacturers to declare the loss values for variable frequency drives at these 8 operating points. Table 5 shows the results of calculating the losses in the electric drive using the SinaSave program at the standard points.

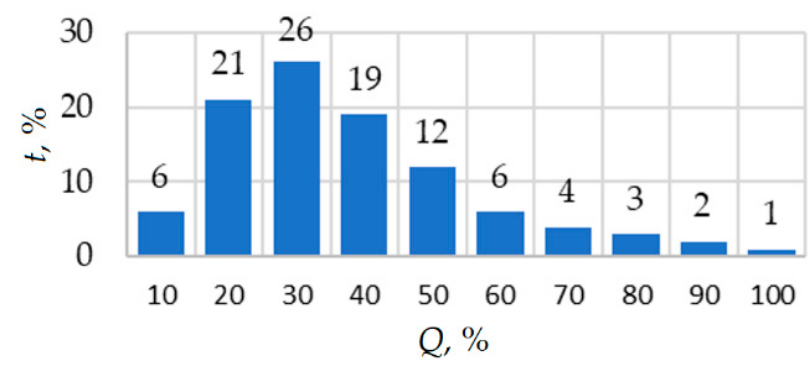

Figure 13. Flow-time diagram.

Table 5. Loss data for the $4 \mathrm{~kW}$ electric drive at the standard loading points.

\begin{tabular}{ccccccccc}
\hline Standard Operating Point & $\mathbf{1}$ & $\mathbf{2}$ & $\mathbf{3}$ & $\mathbf{4}$ & $\mathbf{5}$ & $\mathbf{6}$ & $\mathbf{7}$ & $\mathbf{8}$ \\
\hline$n_{\text {rate drive, } \%} \%$ & 100 & 100 & 50 & 50 & 50 & 0 & 0 & 0 \\
$T_{\text {rate drive, } \%}$ & 100 & 50 & 100 & 50 & 25 & 100 & 50 & 25 \\
$\Delta \mathrm{P}, \mathrm{kW}$ & 0.81 & 0.59 & 0.5 & 0.42 & 0.28 & 0.21 & 0.21 & 0.15 \\
\hline
\end{tabular}

In Table $5, T_{\text {rate drive }}=100 \%=12.93 \mathrm{~N} \cdot \mathrm{m}$ is the rated torque of the electric drive; $n_{\text {rate drive }}=100 \%=2955 \mathrm{rpm}$ is the rated rotational speed of the electric drive.

Using the data from Table 5, the sum of losses in the three pump drives $\triangle \mathrm{P} \Sigma$ at the considered operating points (Tables 2-4) using polynomial interpolation [34,35] were found. The results of this calculation are shown in Table 6. Table 6 also shows the required total mechanical power $\mathrm{P} \Sigma$ from Tables 2-4.

Table 6. Results of the loss calculation in the electric drive of the pump system applying the different regulation methods.

\begin{tabular}{|c|c|c|c|c|c|c|c|c|c|c|c|}
\hline & $Q_{\text {req }}, \%$ & 10 & 20 & 30 & 40 & 50 & 60 & 70 & 80 & 90 & 100 \\
\hline & $Q_{\text {req }}, \mathrm{m}^{3} / \mathrm{h}$ & 12 & 24 & 36 & 48 & 60 & 72 & 84 & 96 & 108 & 120 \\
\hline & Maximum efficiency & 0.24 & 0.27 & 0.33 & 0.43 & 0.93 & 1.05 & 1.18 & 1.33 & 1.75 & 1.88 \\
\hline \multirow{3}{*}{$\Delta \mathrm{P} \Sigma, \mathrm{kW}$} & Maximum reliability & 0.32 & 0.33 & 0.35 & 0.60 & 1.17 & 1.21 & 1.27 & 1.37 & 2.06 & 2.12 \\
\hline & Trade-off & 0.27 & 0.27 & 0.33 & 0.43 & 0.99 & 1.05 & 1.18 & 1.33 & 1.75 & 1.88 \\
\hline & Maximum efficiency & 0.66 & 0.99 & 1.45 & 2.07 & 4.53 & 5.17 & 5.87 & 6.61 & 8.79 & 9.47 \\
\hline \multirow[t]{2}{*}{$\mathbf{P \Sigma}, \mathbf{k W}$} & Maximum reliability & 1.37 & 1.44 & 1.54 & 2.99 & 5.70 & 5.95 & 6.27 & 6.79 & 10.19 & 10.52 \\
\hline & Trade-off & 0.96 & 1.00 & 1.45 & 2.07 & 4.80 & 5.19 & 5.87 & 6.61 & 8.82 & 9.47 \\
\hline
\end{tabular}

Due to the results of calculating the mechanical power and interpolation of losses in the electric drive from Table 6, it can be concluded that the losses in the electric drive also make up a significant part of the energy consumption, about $20-30 \%$, and strongly depend on the operating point of the pump. Therefore, the losses in the electric drive must also be taken into account when calculating the total energy consumption of the pumping system.

Using the results obtained (Tables 2-6), it is possible to calculate the electrical power consumed from the grid $\left(P_{\text {elec }}\right)$, the daily consumed electrical energy $\left(E_{\text {day }}\right)$, the annual consumed electrical energy $\left(E_{\text {year }}\right)$, the annual energy cost $\left(C_{\text {year }}\right)$ and the cost of energy over the entire life cycle of the pumping system $\left(C_{L L C}\right)[36]$ :

$$
P_{\text {elec }}=\mathrm{P} \Sigma+\Delta \mathrm{P} \Sigma ;
$$




$$
\begin{aligned}
E_{\text {day }}= & \frac{t_{\Sigma}}{1000} \cdot \sum_{i=1}^{10}\left(P_{\text {elec }}(i) \cdot \frac{t_{i}}{t_{\Sigma}}\right) ; \\
& E_{\text {year }}=E_{\text {day }} \cdot 365 ; \\
& C_{\text {year }}=E_{\text {year }} G T ; \\
C_{L C C}= & \sum_{k=1}^{w}\left(\frac{C_{\text {year } k}}{(1+[y-p])^{k}}\right),
\end{aligned}
$$

where $\mathrm{P} \Sigma$ is the mechanical power required by the pumps; $\Delta \mathrm{P}$ is the loss in the electric drive; $t_{\Sigma}=24 \mathrm{~h}$ is the whole operating period; $t_{i}$ is the operation time of $i$-th loading point; $G T=0.2036 € / \mathrm{kWh}$ is the applied grid tariffs for non-household consumers for Germany in the second half of 2019 [37]; $C_{\text {year }}$ is the annual electricity cost; $C_{\text {year }}$ is the annual electricity cost for $k$-th year; $y=0.06$ is the interest rate; $p=0.04$ is the expected annual inflation; $w=20$ years is the lifetime of the pump system.

Annual and life cycle cost savings $S_{y e a r}$ and $S_{L C C}$ for a given regulation method compared to the maximum reliability method is calculated as:

$$
\begin{aligned}
& S_{\text {year }}=C_{\text {year max. reliab. }}-C_{\text {year }} ; \\
& S_{L C C}=C_{L C C \text { max. reliab. }}-C_{L C C},
\end{aligned}
$$

where $C_{\text {year max. reliab. }}$ is the annual energy cost with the maximum reliability control; $C_{L C C}$ max. reliab is the lifetime energy cost with the maximum reliability control.

$S_{\text {year }}$ year $S_{L C C}$ percentage are calculated according to:

\begin{tabular}{|c|c|c|c|c|c|c|c|c|c|c|c|}
\hline & $Q_{\text {req }} \%$ & 10 & 20 & 30 & 40 & 50 & 60 & 70 & 80 & 90 & 100 \\
\hline & $Q_{\text {req }}, \mathrm{m}^{3} / \mathrm{h}$ & 12 & 24 & 36 & 48 & 60 & 72 & 84 & 96 & 108 & 120 \\
\hline & Maximum efficiency & 0.90 & 1.27 & 1.78 & 2.50 & 5.47 & 6.22 & 7.04 & 7.94 & 10.53 & 11.35 \\
\hline$P_{\text {elec }}, \mathrm{kW}$ & Maximum reliability & 1.70 & 1.77 & 1.89 & 3.59 & 6.87 & 7.16 & 7.53 & 8.16 & 12.25 & 12.64 \\
\hline & Trade-off & 1.23 & 1.28 & 1.78 & 2.50 & 5.79 & 6.24 & 7.04 & 7.94 & 10.57 & 11.35 \\
\hline
\end{tabular}

$$
\begin{aligned}
& S_{\text {year }}=100 \% \cdot\left(C_{\text {year max. reliab. }}-C_{\text {year }}\right) / C_{\text {year max. reliab }} ; \\
& S_{L C C}=100 \% \cdot\left(C_{L C C \text { max. reliab. }}-C_{L C C}\right) / C_{L C C \text { max. reliab }},
\end{aligned}
$$

Tables 7 and 8 show the calculation results based on Equations (10)-(17).

Table 7. Results of the electric power calculation in the pump system at the different regulation methods.

Table 8. Results of the lifetime electricity costs in the pump system at the different regulation methods.

\begin{tabular}{cccc}
\hline Control Method & Maximum Efficiency & Maximum Reliability & Trade-Off \\
\hline$E_{\text {day }}, \mathrm{kWh}$ & 75.16 & 91.63 & 76.70 \\
$E_{\text {year }}, \mathrm{kWh}$ & 27,433 & 33,445 & 27,980 \\
$C_{\text {year }}, €$ & 5585 & 6809 & 5697 \\
$S_{\text {year }}, €$ & 1224 & - & 1112 \\
$S_{\text {year. }} \%$ & 18.0 & - & 16.3 \\
$C_{L L C}, \mathrm{k} €$ & 91.3 & 111.3 & 93.1 \\
$S_{L L C}, \mathrm{k} €$ & 20.0 & - & 18.2 \\
$S_{L L C}, \%$ & 18.0 & - & 16.3 \\
\hline
\end{tabular}

\section{Discussion and Results}

From the results obtained, as shown by the diagram in Figure 14, it can be concluded that the highest electrical power consumption is required for maximum reliability control under all considered load conditions. When applying trade-off regulation, a significant increase in electrical power consumption is required only at a flow rate of $10 \%$ and $50 \%$. 


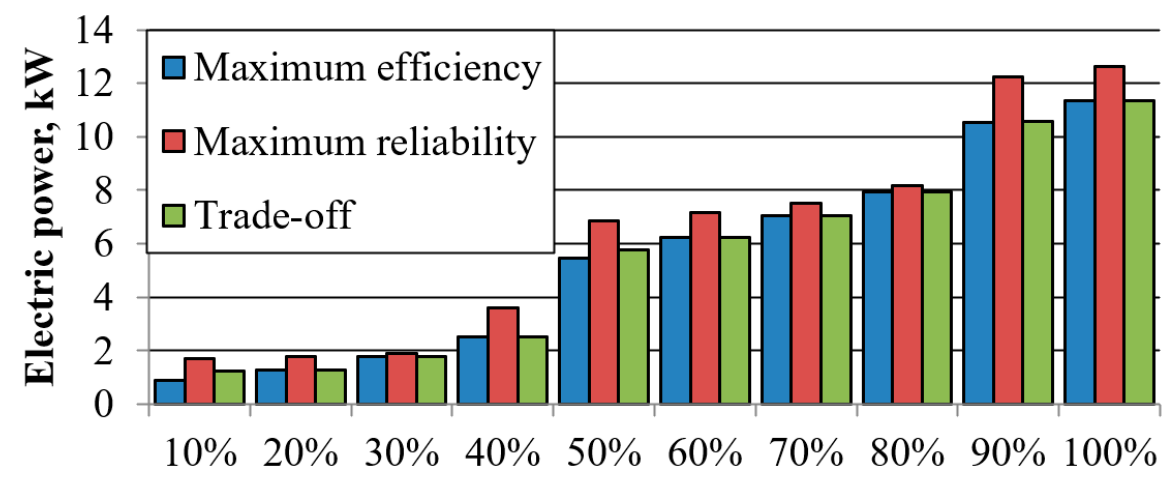

Flow rate, $\%$

Figure 14. Electric power depending on flow rate and regulation method.

Calculation of long-term energy indicators also confirms these conclusions. Figure 15 shows that the reduction in lifetime energy consumption compared to the maximum reliability method differs only slightly in cases of maximum efficiency regulation and tradeoff regulation (18\% and $16.3 \%$, respectively). At the same time, the trade-off method allows all pump operating points to be kept within the POR (Figure 12), which will significantly increase the reliability of the pumping system.

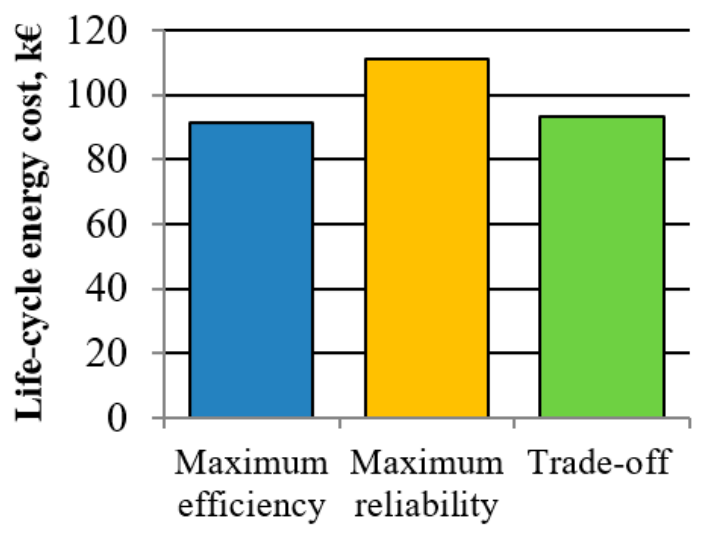

(a)

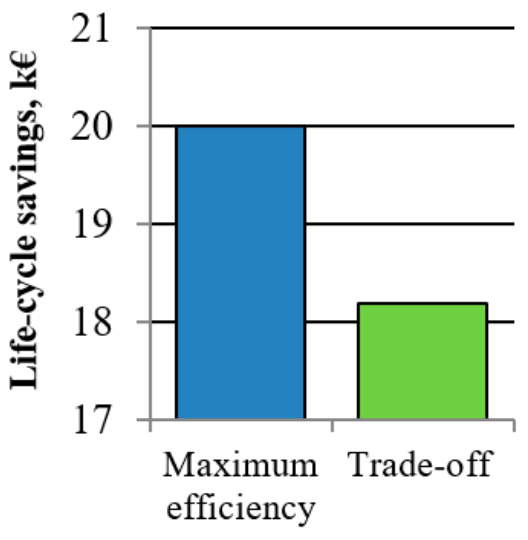

(b)

Figure 15. Energy savings: (a) Life-cycle energy cost depending on the regulation method; (b) life-cycle savings depending on the regulation.

\section{Conclusions}

In this paper, an analysis of the energy consumption of a pumping system consisting of three $4 \mathrm{~kW}$ pumps operating in parallel is carried out, considering the reliability constraints. One $4 \mathrm{~kW}$ variable frequency induction motor and two $4 \mathrm{~kW}$ fixed frequency induction motors are used in the drive of the pumping system.

Energy consumption of the system when three methods of flowrate control regulation are applied is compared: with minimum power consumption, with maximum reliability and trade-off regulation. Comparison of energy consumption in the considered cases shows that energy consumption is $18 \%$ higher with maximum reliability regulation compared to minimum energy consumption regulation without any reliability constraints. At the same time, when trade-off regulation is applied, the power consumption is only $2.3 \%$ higher. It can be concluded that the proposed trade-off regulation is promising in terms of minimizing the overall lifetime costs of the considered multi-pump single-drive system when both energy costs and maintenance and repair costs are considered. 
In future works, it is planned to simulate the dynamics and select a controller for the multi-pump system under consideration when applying the proposed trade-off regulation principle.

Author Contributions: Conceptual approach, S.O. and V.P.; data curation V.D. and V.K.; software S.O. and V.K.; calculations and modeling, S.O., V.K. and V.P.; writing of original draft S.O., V.D., V.K. and V.P.; visualization, S.O. and V.K.; review and editing, S.O., V.D., V.K. and V.P. All authors have read and agreed to the published version of the manuscript.

Funding: The work was partially supported by the Ministry of Science and Higher Education of the Russian Federation (through the basic part of the government mandate, Project No. FEUZ-2020-0060).

Institutional Review Board Statement: Not applicable.

Informed Consent Statement: Not applicable.

Data Availability Statement: Data is contained within the article.

Acknowledgments: The authors thank the editors and reviewers for careful reading and constructive comments.

Conflicts of Interest: The authors declare no conflict of interest.

\section{References}

1. Shankar, V.K.A.; Umashankar, S.; Paramasivam, S.; Hanigovszki, N. A comprehensive review on energy efficiency enhancement initiatives in centrifugal pumping system. Appl. Energy 2016, 181, 495-513. [CrossRef]

2. Frenning, L. (Ed.) Pump Life Cycle Costs: A Guide to LCC Analysis for Pumping Systems, Executive Summary; Hydraulic Institute: Parsippany, NJ, USA; Europump: Brussels, Belgium; Office of Industrial Technologies Energy Efficiency and Renewable Energy, U.S. Department of Energy: Washington, DC, USA, 2001; pp. 1-19. Available online: https://www.energy.gov/sites/prod/files/ 2014/05/f16/pumplcc_1001.pdf (accessed on 5 April 2021).

3. Lai, Z.; Li, Q.; Zhao, A.; Zhou, W.; Xu, H.; Wu, D. Improving Reliability of Pumps in Parallel Pump Systems Using Particle Swam Optimization Approach. IEEE Access 2020, 8, 58427-58434. [CrossRef]

4. Barringer, H.P. A life cycle cost summary. In Proceedings of the International Conference of Maintenance Societies, Perth, Australia, 20-23 May 2003; pp. 20-23.

5. ANSI HI 9.6.3-2012. Rotodynamic Centrifugal and Vertical Pump. Guideline for Allowable Operating Region. Hydraulic Institute Standards. Available online: http:/ / www.reliability-centred-maintenance.com/articles/LifeCycleCostSummary.pdf (accessed on 5 April 2021).

6. Lai, Z.; Wu, P.; Yang, S.; Wu, D. A Control Method to Balance the Efficiency and Reliability of a Time-Delayed Pump-Valve System. Math. Probl. Eng. 2016, 2016, 5898209. [CrossRef]

7. Oshurbekov, S.; Kazakbaev, V.; Prakht, V.; Dmitrievskii, V.; Gevorkov, L. Energy Consumption Comparison of a Single VariableSpeed Pump and a System of Two Pumps: Variable-Speed and Fixed-Speed. Appl. Sci. 2020, 10, 8820. [CrossRef]

8. Wen, Y.; Zhang, X.; Wang, P. The Relationship between the Maximum Efficiency and the Flow of Centrifugal Pumps in Parallel Operation. J. Press. Vessel Technol. 2010, 132, 034501. [CrossRef]

9. Olszewski, P. Genetic optimization and experimental verification of complex parallel pumping station with centrifugal pumps. Appl. Energy 2016, 178, 527-539. [CrossRef]

10. Pandey, S.; Singh, R.P.; Mahar, P.S. Optimal Pipe Sizing and Operation of Multistage Centrifugal Pumps for Water Supply. J. Pipeline Syst. Eng. Pract. 2020, 11, 04020007. [CrossRef]

11. Jia, M.; Zhang, J.; Xu, Y. Optimization Design of Industrial Water Supply Pump Station Considering the Influence of Atmospheric Temperature on Operation Cost. IEEE Access 2020, 8, 161702-161712. [CrossRef]

12. Luo, Y.; Xiong, Z.; Sun, H.; Guo, Y. Research on energy-saving operation control model of the multi-type configuration centrifugal pump system with single invert. Adv. Mech. Eng. 2017, 9, 1-10. [CrossRef]

13. Sike, H.; Xuejing, J.; Huifen, G. Optimization of the Number of Multiple Pumps Running Simultaneously in Open Cycle Cooling Water System in Power Plant. Energy Procedia 2012, 17, 1161-1168. [CrossRef]

14. Bakman, I.; Gevorkov, L.; Vodovozov, V. Predictive control of a variable-speed multi-pump motor drive. In Proceedings of the 2014 IEEE 23rd International Symposium on Industrial Electronics (ISIE), Istanbul, Turkey, 1-4 June 2014; pp. 1409-1414. [CrossRef]

15. Wu, P.; Lai, Z.; Wu, D. Optimization Research of Parallel Pump System for Improving Energy Efficiency. J. Water Resour. Plan. Manag. 2014, 141. [CrossRef]

16. Viholainen, J.; Tamminen, J.; Ahonen, T.; Ahola, J.; Vakkilainen, E.; Soukka, R. Energy-efficient control strategy for variable speed-driven parallel pumping systems. Energy Effic. 2012, 6, 495-509. [CrossRef] 
17. Oshurbekov, S.; Kazakbaev, V.; Prakht, V.; Dmitrievskii, V.; Gevorkov, L. Extending Pump Unit Service Life Using Combined Pump Control. In Proceedings of the 2021 28th International Workshop on Electric Drives: Improving Reliability of Electric Drives (IWED), Moscow, Russia, 27-29 January 2021; pp. 1-6. [CrossRef]

18. Saggewiss, G.; Kotwitz, R.; McIntosh, D. AFD synchronizing applications: Identifying potential methods and benefits. In Proceedings of the 2001 Petroleum and Chemical Industry Technical Conference (Cat. No.01CH37265), Toronto, ON, Canada, 26 September 2001; pp. 83-89. [CrossRef]

19. Automation Pump, Pump Genius. Product Description, WEG. Document Code: 50059602, Revision 04. January 2019. Available online: https:/ / static.weg.net/medias/downloadcenter/hb3/hda/WEG-pump-genius-50059602-brochure-en.pdf (accessed on 5 April 2021).

20. Amirahmadi, A.; Rafiei, M.; Tehrani, K.; Griva, G.; Batarseh, I. Optimum Design of Integer and Fractional-Order PID Controllers for Boost Converter Using SPEA Look-up Tables. J. Power Electron. 2015, 15, 160-176. [CrossRef]

21. Rajesh, R. Optimal tuning of FOPID controller based on PSO algorithm with reference model for a single conical tank system. SN Appl. Sci. 2019, 1, 758. [CrossRef]

22. Bhamre, P.; Kadu, C. Design of a smith predictor based fractional order PID controller for a coupled tank system. In Proceedings of the 2016 International Conference on Automatic Control and Dynamic Optimization Techniques (ICACDOT), Pune, India, 9-10 September 2016; pp. 705-708. [CrossRef]

23. Tepljakov, A.; Petlenkov, E.; Belikov, J. Gain and order scheduled fractional-order PID control of fluid level in a multi-tank system. In Proceedings of the ICFDA'14 International Conference on Fractional Differentiation and Its Applications 2014, Catania, Italy, 23-25 June 2014; pp. 1-6. [CrossRef]

24. Ismail, M.; Bendary, A. FOPID Controller Based AC Pump Supplied from PV Standalone Source Tuned using Fuzzy Logic Type 2. Indones. J. Electr. Eng. Comput. Sci. 2016, 4, 10-19. [CrossRef]

25. Wu, X.; Xu, Y.; Liu, J.; Lv, C.; Zhou, J.; Zhang, Q. Characteristics Analysis and Fuzzy Fractional-Order PID Parameter Optimization for Primary Frequency Modulation of a Pumped Storage Unit Based on a Multi-Objective Gravitational Search Algorithm. Energies 2020, 13, 137. [CrossRef]

26. Stoffel, B. Assessing the Energy Efficiency of Pumps and Pump Units. Background and Methodology, 1st ed.; Elsevier: Amsterdam, The Netherlands, 2015; pp. 8, 9, 109, 113. [CrossRef]

27. NM, NMS. Close Coupled Centrifugal Pumps with Flanged Connections; Catalogue; Calpeda: Montorso Vicentino, Italy, 2018; Available online: https://www.calpeda.com/system/pdf/catalogue_en_50hz.pdf (accessed on 5 April 2021).

28. Dutta, N.; Palanisamy, K.; Subramaniam, U.; Padmanaban, S.; Holm-Nielsen, J.B.; Blaabjerg, F.; Almakhles, D.J. Identification of Water Hammering for Centrifugal Pump Drive Systems. Appl. Sci. 2020, 10, 2683. [CrossRef]

29. Tripathy, A.K.; Nambiar, P.; Pereira, A.; D'souza, S.; Rodrigues, L.; D'souza, A.; D'souza, B.; D'mello, B. Pressure surge analysis in pump systems. In Proceedings of the 2015 International Conference on Technologies for Sustainable Development (ICTSD), Mumbai, India, 4-6 February 2015; pp. 1-5. [CrossRef]

30. SINAMICS G120C. The Compact and Versatile Drive with Optimum Functionality. Available online: https://assets.new.siemens. com/siemens/assets/api/uuid:2c65250d-a8fc-4e40-bbb3-550543d54a1f/version:1573939918/sinamics-g120c-brochure.pdf (accessed on 5 April 2021).

31. SIMOTICS GP, SD, XP, DP Low-Voltage Motors Catalog. Available online: https:/ / cache.industry.siemens.com/dl/files/197/10 9749197/att_955119/v1/simotics-gp-sd-xp-dp-catalogue-d-81-1-en-2018.pdf (accessed on 5 April 2021).

32. Siemens. SinaSave. Available online: https://www.sinasave.siemens.com/\#/ru/pump (accessed on 5 April 2021).

33. IEC. Adjustable Speed Electrical Power Drive Systems_Part 9-2: Ecodesign for Power Drive Systems, Motor Starters, Power Electronics and Their Driven Applications-Energy Efficiency Indicators for Power Drive Systems and Motor Starters; IEC 61800-9-2/Ed1; IEC: Geneva, Switzerland, 2017.

34. Kazakbaev, V.; Prakht, V.; Dmitrievskii, V.; Ibrahim, M.N.; Oshurbekov, S.; Sarapulov, S. Efficiency Analysis of Low Electric Power Drives Employing Induction and Synchronous Reluctance Motors in Pump Applications. Energies 2019, 12, 1144. [CrossRef]

35. Safin, N.; Kazakbaev, V.; Prakht, V.; Dmitrievskii, V.; Sarapulov, S. Interpolation and analysis of the efficiency of a synchronous reluctance electric drive at various load points of a fan profile. In Proceedings of the 25th International Workshop on Electric Drives: Optimization in Control of Electric Drives (IWED 2018), Moscow, Russia, 31 January-2 February 2018; pp. 1-5. [CrossRef]

36. Goman, V.; Oshurbekov, S.; Kazakbaev, V.; Prakht, V.; Dmitrievskii, V. Energy Efficiency Analysis of Fixed-Speed Pump Drives with Various Types of Motors. Appl. Sci. 2019, 9, 5295. [CrossRef]

37. Eurostat Data for the Industrial Consumers in Germany. Available online: http:/ / ec.europa.eu/eurostat/statistics-explained/ index.php/Electricity_price_statistics\#Electricity_prices_for_industrial_consumers (accessed on 5 April 2021). 